\title{
Decentralized Caching Schemes and Performance Limits in Two-layer Networks
}

\author{
Lin Zhang, Zhao Wang, Ming Xiao, Senior Member, IEEE, \\ Gang Wu, Member, IEEE, Ying-Chang Liang, Fellow, IEEE, \\ and Shaoqian Li, Fellow, IEEE
}

\begin{abstract}
We study the decentralized caching scheme in a two-layer network, which includes a sever, multiple helpers, and multiple users. Basically, the proposed caching scheme consists of two phases, i.e, placement phase and delivery phase. In the placement phase, each helper/user randomly and independently selects contents from the server and stores them into its memory. In the delivery phase, the users request contents from the server, and the server satisfies each user through a helper. Different from the existing caching scheme, the proposed caching scheme takes into account the pre-stored contents at both helpers and users in the placement phase to design the delivery phase. Meanwhile, the proposed caching scheme exploits index coding in the delivery phase and leverages multicast opportunities, even when different users request distinct contents. Besides, we analytically characterize the performance limit of the proposed caching scheme, and show that the achievable rate region of the proposed caching scheme lies within constant margins to the information-theoretic optimum. In particular, the multiplicative and additive factors are carefully sharpened to be $\frac{1}{48}$ and 4 respectively, both of which are better than the state of arts. Finally, simulation results demonstrate the advantage of the proposed caching scheme compared with the state of arts.
\end{abstract}

Index Terms-Achievable rate region, cross-layer caching, decentralized coded caching, hybrid scheme, two-layer networks

\section{INTRODUCTION}

Video-on-demand ( $\mathrm{VoD})$ and Internet of Things (IoT) are envisioned to generate massive Internet traffic in the next few years and inevitably causes network congestions [2][6]. To deal with this issue, the content caching is proposed by utilizing the storage capacities of network nodes [7][11]. In particular, if some contents are pre-stored in a local

Copyright (c) 2015 IEEE. Personal use of this material is permitted. However, permission to use this material for any other purposes must be obtained from the IEEE by sending a request to pubs-permissions@ieee.org.

L. Zhang is with the Key Laboratory on Communications, and also with the Center for Intelligent Networking and Communications (CINC), University of Electronic Science and Technology of China (UESTC), Chengdu, China (email: linzhang1913@gmail.com).

Z. Wang is with Ericsson Research, Stockholm, Sweden (email: zhao.wang@ericsson.com).

M. Xiao is with the School of Electrical Engineering, Royal Institute of Technology (KTH), Stockholm, Sweden (email: mingx@kth.se).

G. Wu and S. Li are with the Key Laboratory on Communications, University of Electronic Science and Technology of China (UESTC), Chengdu, China (emails: wugang99@uestc.edu.cn and 1sq@uestc.edu.cn).

Y.-C. Liang is with the Center for Intelligent Networking and Communications (CINC), University of Electronic Science and Technology of China (UESTC), Chengdu, China (email: liangyc @ieee.org).

This work was supported in part by National Natural Science Foundation of China under Grants 61571100, 61631005, 61628103, and 61801101.

Part of this paper has been presented in [1] at IEEE CIC/ICCC 2016. storage close to a user, these pre-stored contents can be directly accessed by the user. This avoids unnecessary content deliveries from servers and thus releases network congestions. Apparently, this mechanism is able to offer a local caching gain, which is particularly relevant when the local storage is large.

Recently, Maddah-Ali \& Niesen (MAU) introduced index coding into a singer-layer content caching network [12], [13], which includes a server and multiple users. In particular, MAU proposed a centralized caching scheme in [12] and a decentralized caching scheme in [13], respectively (We name them as MAU-Centralized scheme and MAU-Decentralized scheme respectively hereafter.). The basic idea of the centralized and decentralized caching schemes is as follows: By viewing the pre-stored contents as the side-information, a coded delivery can be designed to create a single-layer multicast opportunity (SMO), even when users request distinct contents. With this idea, both caching schemes are able to reduce the traffic load of the network and reveal coding gains. It is worth noting that both coding gains are shown to be proportional to the aggregation of all the local storage capacities. Thus, the content caching with index coding is able to leverage both local caching gain and global coding gain.

Due to the centralized processing, the MAU-Centralized scheme provides a larger coding gain than the MAUDecentralized scheme. Nevertheless, the MAU-Centralized scheme is sensitive to the instantaneous network profile and needs redesigns once the instantaneous network profile changes, while the MAU-Decentralized scheme is independent from the instantaneous network profile and thus is more robust than the MAU-Centralized scheme. It is remarkable that the MAU-Decentralized scheme is proved to be with the same order-optimality as the MAU-Centralized scheme, i.e., the same multiplicative and additive factors.

In practical situations, the network topology is usually tree-like and users have to obtain contents through some intermediate nodes, namely, helpers, from a server. Then, [14] considered a two-layer network and intended to minimize the traffic loads of both layers, i.e., the first layer is from the server to the helpers and the second layer is from each helper to its attached users. In particular, [14] proposed a generalized caching scheme by exploiting both the SMO of each layer and the cross-layer multicast opportunity (CMO) between the server and users. The important observation from [14] is that almost no tension exists between the traffic loads of two layers, such that the loads of both layers can be reduced 
simultaneously. Yet, the problem still remains on whether the traffic loads of both layers can be further reduced, which is both practically and theoretically important. In the rest of the paper, we describe the traffic load with a normalized transmission rate, which will be formally defined later, and we use "traffic load" and "transmission rate" interchangeably. In particular, a large/small transmission rate corresponds to a large/small traffic load.

\section{A. Main contributions}

In this paper, we study the decentralized caching in a twolayer caching network similar to [14]. It is worth noting that, a two-layer caching network can be divided into multiple single-layer caching sub-networks. In particular, the server and the helpers form a single-layer caching sub-network, in which each helper requests multiple contents from the server. Each helper and its attached users form a singlelayer caching sub-network, in which each user requests one content from the associated helper. Besides [12] and [13], advanced caching schemes in a single-layer caching network have been extensively studied in recent years. These advanced caching schemes can be directly applied into each single-layer caching sub-network, if we treat these single-layer caching sub-networks separately. However, the direct application of these advanced caching schemes may be suboptimal in terms of transmission rates in the network. Instead, we focus on the joint caching design of the two layers. To highlight the contributions, we list the main contents of this paper.

- We notice that the pre-stored contents at user's storage do not need to be recovered at the associated helper. This reduces the transmission rate of the first layer and creates a cross-layer caching gain (CCG). Then, we develop an S\&C caching scheme to exploit both the SMO and CCG. Meanwhile, we analytically derive the corresponding transmission rate of each layer with a closed-form expression.

- We propose a hybrid algorithm to exploit the SMO, CMO, and CCG simultaneously. Specifically, we segment the whole network into two parallel sub-networks and combine the S\&C caching scheme and the caching scheme B in [14] in a memory-sharing manner. Meanwhile, we introduce $\alpha, \beta \in[0,1]$ as the memory sharing parameters in the network segmentation, and analytically derive the transmission rate of each layer with a closedform expression as a function of $\alpha$ and $\beta$. Notably, different tuples of $(\alpha, \beta)$ correspond to different caching designs. By comparing the closed-form expressions with those in [14], we show that the transmission rate of the first layer can be reduced without increasing the transmission rate of the second layer for any $(\alpha, \beta)$. It is worth noting that, the reduction of the transmission rate in the first layer is of significant importance, since the maximum traffic load at a server in the first layer is usually the bottleneck of the network capacity and the load reduction at the server enables the server to support more helpers/users and thus boosts the network capacity.
- We analytically optimize $(\alpha, \beta)$ and obtain the performance limit of the hybrid caching scheme as follows,

$$
\mathcal{R}^{H}\left(M_{1}, M_{2}\right) \subset \mathcal{R}\left(M_{1}, M_{2}\right) \subset c_{1} \mathcal{R}^{H}\left(M_{1}, M_{2}\right)-c_{2},
$$

where $M_{1}$ and $M_{2}$ are the normalized storage sizes at helpers and users respectively, $\mathcal{R}^{H}$ is the achievable rate region with the proposed hybrid caching scheme and $\mathcal{R}$ is the information-theoretic (optimal) rate region. In particular, the multiplicative and additive factors are carefully quantified to be $c_{1}=\frac{1}{48}$ and $c_{2}=4$ respectively, both of which are better than those in [14].

Compared with [14], the contribution of the paper is fourfold. Firstly, we propose a hybrid caching scheme to reduce the traffic load in a two-layer caching network. Secondly, we provide optimized tuples of $(\alpha, \beta)$ for different settings of the network. Thirdly, we elaborate a better quantification of the gaps between the achievable rates and the informationtheoretic minimum rates. Finally, by adopting the overall traffic load in the network as the metric in simulations, we demonstrate that the proposed caching scheme is able to effectively reduce the traffic loads in different network settings compared with the caching scheme in [14]. Although the motivation of the paper originates from a trivial observation of the scheme in [14], the proposed caching scheme and the associated information-theoretic analysis are not straightforward.

\section{B. Related works}

After [12] and [13], the coded caching problems are widely studied from various aspects. [15] studied the centralized coded caching and intended to improve the performance of the caching scheme in [12]. In particular, authors in [15] treated the design of caching scheme as a combinatorial problem of optimally labeling the leaves of a directed tree. By developing a novel labeling algorithm, the lower bound of the transmission rate in [12] is significantly improved. [16] proposed a novel coded caching scheme subject to a storage size constraint, and showed the improvement of the required transmission rate compared with the existing approaches when the number of users satisfied some conditions. The coded caching with different storage sizes is studied in [17], [18]. In particular, [17] proposed a new decentralized coded caching scheme and showed the reduction of the required transmission rate compared with the existing results when the number of users is larger than the number of files. [18] proposed an optimization framework for cache placement and delivery schemes, which explicitly accounted for different storage sizes and characterized the optimal caching scheme when the overall users storage size is no larger than the storage size of the file server.

[19] and [20] considered the randomness of user demands and intended to reduce the average transmission rate. In particular, [19] partitioned files with similar request probabilities into a group, and applied the caching scheme in [13] to each group. However, the scheme in [19] cannot guarantee rate order-optimality in all regimes of the system parameters. Then, [20] optimized the caching scheme in [19] and proposed a new 


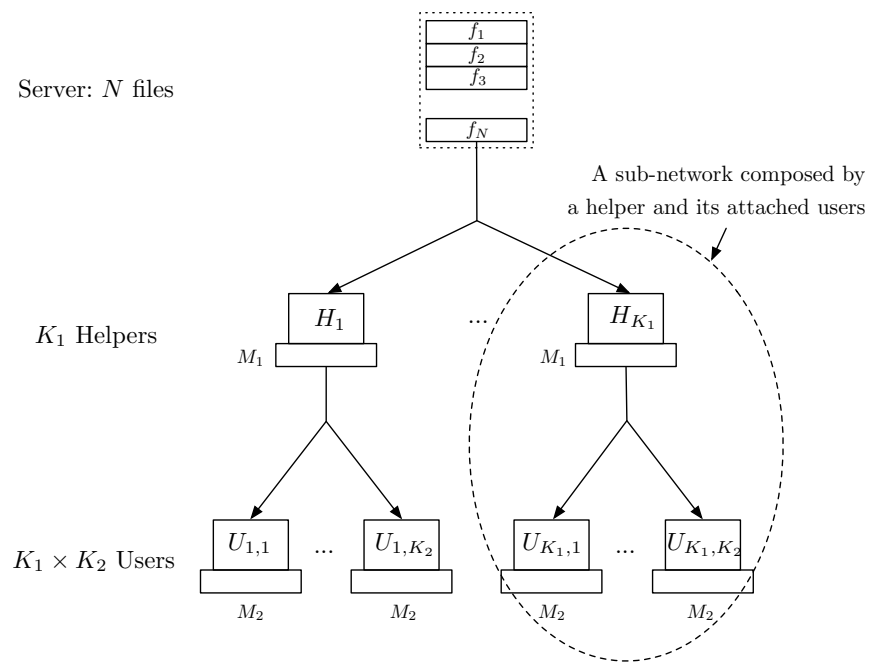

Fig. 1. A two-layer network with one server hosting $N$ files, $K_{1}$ helpers with normalized memory size $M_{1}$, i.e., $M_{1} F$ bits, and $K_{1} K_{2}$ users with normalized memory size $M_{2}$, i.e., $M_{2} F$ bits. Each helper is dedicated for helping $K_{2}$ users. The straight line between two nodes represents the wireless channel. Similar to [10], we assume that there is no interference among different sub-networks.

caching approach with the theoretically analysis of its orderoptimality. [21] considered different file sizes and studied the performance limits of coded caching. In particular, [21] derived a new lower bound and an achievable upper bound for the worst case transmission rate.

Besides, [22]-[24] considered multiple demands from each user and [25], [26] investigated online caching schemes. Other researches include caching problems in heterogeneous networks [27], [28], device-to-device (D2D) assisted caching [29], [30], caching in the finite length regime [31]-[33], security in caching networks [34]-[36], and so on.

\section{SySTEM MODEL}

We consider a two-layer caching network as shown in Fig. 1 which includes a server, $K_{1}$ helpers, and $K_{1} K_{2}$ users. In this network, the server is connected to $K_{1}\left(K_{1} \geq 2\right)$ helpers $H_{i}\left(i \in\left[1: K_{1}\right]\right)$, and each helper is exclusively connected to $K_{2}\left(K_{2} \geq 2\right)^{*}$ users $U_{i, j}\left(j \in\left[1: K_{2}\right]\right)$. In particular, the server hosts $N$ files $\mathcal{S}=\left\{f_{n}: n \in[1: N]\right\}$, each of which has $F$ bits. The storage capacities of each helper and each user are $M_{1} F$ bits and $M_{2} F$ bits $\left(M_{1}, M_{2} \leq N\right)$, respectively. For convenience, we denote $M_{1}=\frac{M_{1} F}{F}$ and $M_{2}=\frac{M_{2} F}{F}$ as the normalized storage capacities of each helper and each user, respectively. The content caching in the network has two orthogonal phases, i.e., placement phase and delivery phase. In the placement phase, $M_{1} F$ bits are pre-stored in the storage of each helper and $M_{2} F$ bits are pre-stored in the storage of each user. In the delivery phase, each user requests a specific file. If some contents of the requested file are in the local

${ }^{*}$ For $K_{1}=1$ or $K_{2}=1$, the two-layer network can be reduced to a single-layer network. Thus, we study the two-layer network with $K_{1} \geq 2$ and $K_{2} \geq 2$ in this paper.

${ }^{\dagger}$ For $M_{1}>N$, it is straightforward to reduce the two-layer system to a single-layer system. For $M_{2}>N$, each user is able to cache all the files to the local storage. storage, the user directly accesses these contents. Otherwise, the user requests these contents from the associated helper and/or server.

It is worth noting that the transmission rates in the network (including the transmission rate from the server to the helpers and the transmission rate from each helper to its attached users) are determined by the requested files of users and the pre-stored (cached) contents in each helper/user. Then, different helpers may have different transmission rates depending on the requested files of users and the pre-stored (cached) contents in each helper/user. Similar to [10], we are interested in the worst case in which different users request distinct files, and focus on the decentralized caching scheme in which each helper/user caches contents randomly and independently f $^{\text {. }}$ Thus, different helpers have an identical (worst-case) rate. In particular, by denoting $L_{1}$ as the number of the delivered bits from the server to the helpers and denoting $L_{2}$ as the number of the delivered bits from each helper to its attached users, we define $R_{1}=\frac{L_{1}}{F}$ and $R_{2}=\frac{L_{2}}{F}$ as the normalized transmission rate from the server and each helper, respectively.

\section{S\&C CACHING SCHEME: EXPLOITING BOTH SMO AND $\mathrm{CCG}$}

In this section, we propose an $\mathrm{S} \& \mathrm{C}$ caching scheme to exploit the SMO from the server to the helpers, the SMO from each helper to its attached users, and the CCG between the helpers and the users. In what follows, we first present the principle and results of this scheme, and then provide the placement algorithm and delivery algorithm, respectively.

\section{A. Principle and results of the $S \& C$ caching scheme}

In the considered two-layer caching network, if we directly apply the MAU-Decentralized scheme in each layer, each requested file will be resolved at both the user and the associated helper. In fact, if we jointly take the pre-stored contents at the helper and the user into account, the prestored contents at the user do not need to be recovered at the associated helper. Therefore, it is possible to reduce the transmission rate with the CCG between helpers and users.

Lemma 1: For $M_{1} \leq N$ and $M_{2} \leq N$, with the $\mathrm{S} \& \mathrm{C}$ caching scheme, the transmission rate from the server to the helpers is

$$
R_{1}^{S \& C}=K_{2}\left(1-\frac{M_{2}}{N}\right) \gamma\left(\frac{M_{1}}{N}, K_{1}\right),
$$

and the transmission rate from each helper to its attached users is

$$
R_{2}^{S \& C}=\gamma\left(\frac{M_{2}}{N}, K_{2}\right),
$$

$\ddagger$ Note that, although centralized caching may further reduce the traffic load in the two-layer network, it is not straightforward to apply centralized caching into the two-layer network. The reasons are as follows: On one hand, in the single-layer caching network, the centralized caching in [8] is different from the decentralized caching in [9] in terms of content placement schemes, content delivery schemes, and information-theoretic quantifications. On the other hand, the two-layer network is more complicated than the single-layer network, since joint optimizations (including content placement schemes, content delivery schemes, and information-theoretic quantifications) between the two layers need to be conducted. The centralized caching is beyond the scope of the paper. 
where

$\gamma\left(\frac{M}{N}, K\right) \triangleq K\left(1-\frac{M}{N}\right) \frac{N}{K M}\left(1-\left(1-\frac{M}{N}\right)^{K}\right) \geq 0$.

Remark 1 (Performance comparison with [14]): In the decentralized caching scheme A of [14], the MAU-Decentralized scheme is directly applied in each individual layer. Accordingly, the transmission rate from the server to the helpers is $R_{1}^{A}=K_{2} \gamma\left(\frac{M_{1}}{N}, K_{1}\right)$ and the transmission rate from each helper to its attached users is $R_{2}^{A}=\gamma\left(\frac{M_{2}}{N}, K_{2}\right)$. By comparing $R_{1}^{S \& C}$ with $R_{1}^{A}$, we have $R_{1}^{S \& C}=R_{1}^{A}\left(1-\frac{M_{2}}{N}\right)$, which is strictly smaller than $R_{1}^{A}$ due to $1-\frac{M_{2}}{N}<1$. Besides, we observe that $R_{2}^{S \& C}$ is equal to $R_{2}^{A}$. Therefore, with the $\mathrm{S} \& \mathrm{C}$ caching scheme, the transmission rate in the first layer can be reduced without increasing the transmission rate in the second layer compared with the decentralized caching scheme $\mathrm{A}$ in [14].

\section{B. Placement and Delivery of the $S \& C$ scheme}

In this part, we present the detailed placement and delivery methods of the S\&C scheme.

1) Placement at helpers and users: For the placement at helpers, each helper randomly and independently selects $\frac{M_{1} F}{N}$ bits from each file $n \in[1: N]$ and stores them into its storage. Similarly, for the placement at users, each user randomly and independently selects $\frac{M_{2} F}{N}$ bits from each file $n \in[1: N]$ and stores them into its storage.

2) Delivery from the server to helpers: To begin with, let $d_{i, j}$ denote the requested file index of $U_{i, j}$, let $V_{d_{i, j}, S}$ denote the contents of file $f_{d_{i, j}}$ that are exclusively cached at the nodes in set $S$. From the result in [13], the server needs to transmit $\left\{\oplus_{i \in S_{1}^{H}} V_{d_{i, j}, S_{1}^{H} \backslash i}: S_{1}^{H} \subset\left[1: K_{1}\right],\left|S_{1}^{H}\right|=\right.$ $\left.s_{1}, s_{1} \in\left[1: K_{1}\right], j \in\left[1, K_{2}\right]\right\}$, where $S_{1}^{H}$ denotes the set of helper indexes, such that each helper can recover the requested files of its attached users. Since the contents are randomly and independently placed at helpers and users, each bit in $V_{d_{i, j}, S_{1}^{H} \backslash i}$ is cached in user $U_{i, j}$ with probability $\frac{M_{2}}{N}$. As a result, we can divide each $V_{d_{i, j}, S_{1}^{H} \backslash i}$ in $\left\{\oplus_{i \in S_{1}} V_{d_{i, j}, S_{1}^{H} \backslash i}\right.$ : $\left.S_{1}^{H} \subset\left[1: K_{1}\right],\left|S_{1}^{H}\right|=s_{1}, s_{1} \in\left[1: K_{1}\right], j \in\left[1, K_{2}\right]\right\}$ into two parts, i.e.,

$$
V_{d_{i, j}, S_{1}^{H} \backslash i}=\left\{V_{d_{i, j}, S_{1}^{H} \backslash i}^{U_{i, j}}, V_{d_{i, j}, S_{1}^{H} \backslash i}^{\bar{U}_{i, j}}\right\},
$$

where $V_{d_{i, j}, S_{1}^{H} \backslash i}^{U_{i, j}}$ denotes the part that is cached in user $U_{i, j}$ and $V_{d_{i, j}, S_{1}^{H} \backslash i}^{\bar{U}_{i, j}}$ is the part that is not cached in user $U_{i, j}$. Then, we have

$$
\left|V_{d_{i, j}, S_{1}^{H} \backslash i}^{U_{i, j}}\right| \approx \frac{M_{2}}{N}\left|V_{d_{i, j}, S_{1}^{H} \backslash i}\right|
$$

and

$$
\left|V_{d_{i, j}, S_{1}^{H} \backslash i}^{\bar{U}_{i, j}}\right| \approx\left(1-\frac{M_{2}}{N}\right)\left|V_{d_{i, j}, S_{1}^{H} \backslash i}\right| .
$$

In particular, when the file size $F$ is large enough, the approximations in (5) and (6) can be replaced by equalities according to the law of large numbers.

Since $V_{d_{i, j}, S_{1}^{H} \backslash i}^{U_{i, j}}$ can be locally accessed by user $U_{i, j}$, helper $H_{i}$ does not need to recover $V_{d_{i, j}, S_{1}^{H} \backslash i}^{U_{i, j}}$. In other words, the server only needs to transmit $\left\{\oplus_{i \in S_{1}^{H}} V_{d_{i, j}, S_{1}^{H} \backslash i}^{\bar{U}_{i, j}}: S_{1}^{H} \subset[1\right.$ : $\left.\left.K_{1}\right],\left|S_{1}^{H}\right|=s_{1}, s_{1} \in\left[1: K_{1}\right], j \in\left[1, K_{2}\right]\right\}$, such that helper $H_{i}$ obtains all the requested subfiles in $f_{d_{i, j}}^{\bar{H}_{i}, \bar{U}_{i, j}}=$ $\left\{V_{d_{i, j}, S_{1}^{H} \backslash i}^{\bar{U}_{i, j}}: S_{1}^{H} \subset\left[1: K_{1}\right], i \in S_{1},\left|S_{1}^{H}\right|=s_{1}, s_{1} \in[1:\right.$ $\left.\left.K_{1}\right], j \stackrel{1}{\in}\left[1, K_{2}\right]\right\}$ that are pre-stored in neither of helper $H_{i}$ and users. Recall that, the transmission rate from the server with the scheme in [13] is $R_{1}^{A}=K_{2} \gamma\left(M_{1}, K_{1}\right)$. Thus, the transmission rate from the server with the proposed approach is $K_{2}\left(1-\frac{M_{2}}{N}\right) \gamma\left(\frac{M_{1}}{N}, K_{1}\right)=\left(1-\frac{M_{2}}{N}\right) R_{1}^{A}$ as shown in (1).

3) Delivery from helper $H_{i}$ to the attached users: From the result in [13], helper $H_{i}$ needs to transmit $\left\{\oplus_{j \in S_{2}^{U}} V_{d_{i, j}, S_{2}^{U} \backslash j}\right.$ : $\left.S_{2}^{U} \subset\left[1: K_{2}\right],\left|S_{2}^{U}\right|=s_{2}, s_{2} \in\left[1: K_{2}\right]\right\}$, where $S_{2}^{U}$ denotes the set of user indexes attached to helper $H_{i}$, such that each attached user can recover the requested files. Here, we also enable helper $H_{i}$ to transmit $\left\{\oplus_{j \in S_{2}^{U}} V_{d_{i, j}, S_{2}^{U} \backslash j}: S_{2}^{U} \subset\right.$ $\left.\left[1: K_{2}\right],\left|S_{2}^{U}\right|=s_{2}, s_{2} \in\left[1: K_{2}\right]\right\}$ and satisfy its attached users. In what follows, we shall prove that all the contents in $\left\{\oplus_{j \in S_{2}^{U}} V_{d_{i, j}, S_{2}^{U} \backslash j}: S_{2}^{U} \subset\left[1: K_{2}\right],\left|S_{2}^{U}\right|=s_{2}, s_{2} \in\left[1: K_{2}\right]\right\}$ have been pre-stored or recovered by helper $H_{i}$.

According to whether the contents in $V_{d_{i, j}, S_{2}^{U} \backslash j}$ are prestored in helper $H_{i}, V_{d_{i, j}, S_{2}^{U} \backslash j}$ can be divided into two parts, i.e.,

$$
V_{d_{i, j}, S_{2}^{U} \backslash j}=\left\{V_{d_{i, j}, S_{2}^{U} \backslash j}^{H_{i}}, V_{d_{i, j}, S_{2}^{U} \backslash j}^{\bar{H}_{i}}\right\},
$$

where $V_{d_{i, j}, S_{2}^{U} \backslash j}^{H_{i}}$ denotes the part that is pre-stored in helper $H_{i}$ and $V_{d_{i, j}, S_{2}^{U} \backslash j}^{H_{i}}$ is the part that is not pre-stored in helper $H_{i}$. It is clear that $V_{d_{i, j}, S_{2}^{U} \backslash j}^{\bar{H}_{i}}$ is pre-stored in neither of helper $H_{i}$ nor user $U_{i, j}$. Then, $V_{d_{i, j}, S_{2}^{U} \backslash j}^{\bar{H}_{i}}$ must be in $f_{d_{i, j}}^{\bar{H}_{i}, \bar{U}_{i, j}}$, which has been obtained by helper $H_{i}$. Thus, if helper $H_{i}$ transmits $\left\{\oplus_{j \in S_{2}^{U}} V_{d_{i, j}, S_{2}^{U} \backslash j}: S_{2}^{U} \subset\left[1: K_{2}\right],\left|S_{2}^{U}\right|=s_{2}, s_{2} \in\left[1: K_{2}\right]\right\}$, all the requests of its attached users can be satisfied. Recall that, the transmission rate of each helper with the scheme in [13] is $R_{2}^{A}=\gamma\left(\frac{M_{2}}{N}, K_{2}\right)$. Thus, the transmission rate of each helper with the proposed approach is $R_{2}^{S \& C}=R_{2}^{A}=$ $\gamma\left(\frac{M_{2}}{N}, K_{2}\right)$.

\section{HYBRID CACHING IN TWO-LAYER NETWORKS}

In this section, we present a hybrid caching scheme to exploit SMO, CMO, and CCG simultaneously. For convenience, we first introduce the caching scheme B of [14], which exploits CMO. Then, we combine the S\&C caching scheme with the caching scheme B of [14] in a memory-sharing manner and develop the hybrid caching scheme.

\section{A. Caching scheme $B$ in $[14$}

1) Basic principle and results: To exploit the $\mathrm{CMO}$, the MAU-Decentralized caching scheme is directly applied between the server and $K_{1} K_{2}$ users by ignoring the storages of the helpers. Specifically, each user randomly and independently selects $\frac{M_{2} F}{N}$ bits of each file and stores them into its storage in the placement phase. Then, the server conducts MAU-Decentralized delivery to satisfy all the users through the helpers. Note that there is no direct link between the server and the users. Thus, the server first transmits contents to the helpers. Then, each helper forwards the contents, 
which are relevant to the requested files of its attached users. Finally, each user recovers the requested file by combining the forwarded contents of the associated helper and the prestored contents in its own storage. With this caching scheme, the transmission rate from the server to the helpers is

$$
R_{1}^{B}=\gamma\left(\frac{M_{2}}{N}, K_{1} K_{2}\right)
$$

and the transmission rate from each helper to its attached users is

$$
R_{2}^{B}=\gamma\left(\frac{M_{2}}{N}, K_{2}\right)
$$

2) Placement at users: In the placement phase, each user randomly and independently selects $\frac{M_{2} F}{N}$ bits of each file $n \in$ $[1: N]$ and caches them into its storage.

3) Delivery from server to helpers: From the results in [13], the server needs to transmit $\left\{\oplus_{(i-1) K_{2}+j \in S_{3}} V_{d_{i, j}, S_{3} \backslash(i-1) K_{2}+j}: S_{3} \subset\left[1: K_{1} K_{2}\right],\left|S_{3}\right|=\right.$ $\left.s_{3}, s_{3} \in\left[1: K_{1} K_{2}\right]\right\}$, where $S_{3}$ denotes a set of user indexes, such that each user can recover the requested file. Then, the transmission rate from the server to the helpers can be obtained as (8).

4) Delivery from helper $H_{i}$ to the attached users: From the results in [13], if helper $H_{i}$ forwards the contents relevant to the requested files of its attached users, the transmission rate from helper $H_{i}$ to its attached users can be obtained as (9).

\section{B. Hybrid caching scheme}

1) Basic principle and results: Note that, the $\mathrm{S} \& \mathrm{C}$ caching scheme creates both SMO and CCG by utilizing the storage capacity of the helpers, while the decentralized caching scheme $\mathrm{B}$ of [14] creates $\mathrm{CMO}$ by ignoring the storage capacity of each helper. To exploit SMO, CCG, and CMO simultaneously, we divide the whole network into two parrel sub-networks and apply S\&C caching scheme and the decentralized caching scheme B of [14] in a memory-sharing manner. In particular, we split each file into two parts with sizes $\alpha F(0 \leq \alpha \leq 1)$ and $(1-\alpha) F$ bits. Meanwhile, we partition the storage capacity of each user into two parts of normalized storage capacities $\beta M_{2}(0 \leq \beta \leq 1)$ and $(1-\beta) M_{2}$. Then, the first sub-network consists of a server hosting the first fraction $\alpha$ part of each file, $K_{1}$ helpers each with a normalized storage capacity $M_{1}$, and $K_{1} K_{2}$ users each with a normalized storage capacity $\beta M_{2}$. The second sub-network consists of the server hosting the second fraction $1-\alpha$ part of each file, $K_{1}$ helpers each without any storage capacity, and $K_{1} K_{2}$ users each with a normalized storage capacity $(1-\beta) M_{2}$. In this way, we can apply the $\mathbf{S} \& \mathbf{C}$ caching scheme in the first sub-network which delivers the first fraction $\alpha$ part of each file, and apply the CMO caching scheme in the second sub-network which delivers the second fraction $1-\alpha$ part of each file. It is worth noting that, with the hybrid caching scheme, the overall transmission rate of each layer is the sum rate of the layer in two sub-networks.

Lemma 2: For $M_{1} \leq N$ and $M_{2} \leq N$, the transmission rate from the server to the helpers is

$$
\begin{aligned}
R_{1}^{H}(\alpha, \beta)= & \alpha K_{2}\left[\left(1-\frac{\beta M_{2}}{\alpha N}\right)\right]^{+}\left[\gamma\left(\frac{M_{1}}{\alpha N}, K_{1}\right)\right]^{+}+ \\
& (1-\alpha)\left[\gamma\left(\frac{(1-\beta) M_{2}}{(1-\alpha) N}, K_{1} K_{2}\right)\right]^{+}
\end{aligned}
$$

and the transmission rate from each helper to its attached users is

$$
\begin{aligned}
R_{2}^{H}(\alpha, \beta)= & \alpha\left[\gamma\left(\frac{\beta M_{2}}{\alpha N}, K_{2}\right)\right]^{+}+ \\
& (1-\alpha)\left[\gamma\left(\frac{(1-\beta) M_{2}}{(1-\alpha) N}, K_{2}\right)\right]^{+},
\end{aligned}
$$

where $[x]^{+} \triangleq \max \{x, 0\}$.

Proof: $R_{1}^{H}(\alpha, \beta)$ and $R_{2}^{H}(\alpha, \beta)$ can be obtained by applying the $\mathrm{S} \& \mathrm{C}$ caching scheme and the caching scheme $\mathrm{B}$ of [14] in a memory-sharing manner with factor $\alpha$ and $\beta$. The proof is similar to that in [14] and will be omitted for space limitation.

Remark 2: Performance comparison with [14]: It is worth noting that, the generalized caching scheme in [14] is developed by combining the caching scheme A and B therein in a memory-sharing manner, and the proposed hybrid caching scheme is obtained by combining the $\mathrm{S} \& \mathrm{C}$ caching scheme and the caching scheme B in [14]. Recall that, with the S\&C caching scheme, the transmission rate in the first layer can be reduced without increasing the transmission rate in the second layer compared with the caching scheme A in [14]. Thus, for any $(\alpha, \beta)$, the first-layer transmission rate of the hybrid caching scheme is smaller than that of the generalized caching scheme in [14]. In fact, the reduction of the transmission rate in the first layer is of significant importance, since the maximum traffic load at a server in the first layer is usually the bottleneck of the network capacity and the load reduction at the server enables the server to support more helpers/users and thus boosts the network capacity. Meanwhile, the transmission rates in the second layer with both schemes are identical. This is due to the fact that, both the proposed hybrid caching scheme and generalized caching scheme in [14] adopt the single-layer caching scheme in [13] for the content transmission from each helper to the attached users.

2) Performance limit: Clearly, different tuples of $(\alpha, \beta)$ lead to different hybrid caching designs and transmission rates in two layers. In this part, we first optimize $(\alpha, \beta)$ and evaluate the achievable rates by applying the optimized $(\alpha, \beta)$ into (10) and (11). Then, we quantify the gap between the achievable rate region and the information-theoretic (optimal) rate region, after characterizing the differences between the achievable rates and their information-theoretic lower bounds in two layers, respectively.

To begin with, we formally define the achievable rate region of the hybrid caching scheme and the information-theoretic (theoretically optimal) rate region in the following.

Definition 1: For normalized memory size $M_{1}, M_{2} \geq 0$, we define

$\mathcal{R}^{H}\left(M_{1}, M_{2}\right)=\left\{\left(R_{1}^{H}(\alpha, \beta), R_{2}^{H}(\alpha, \beta)\right): \alpha, \beta \in[0,1]\right\}+\mathbb{R}_{+}^{2}$ 


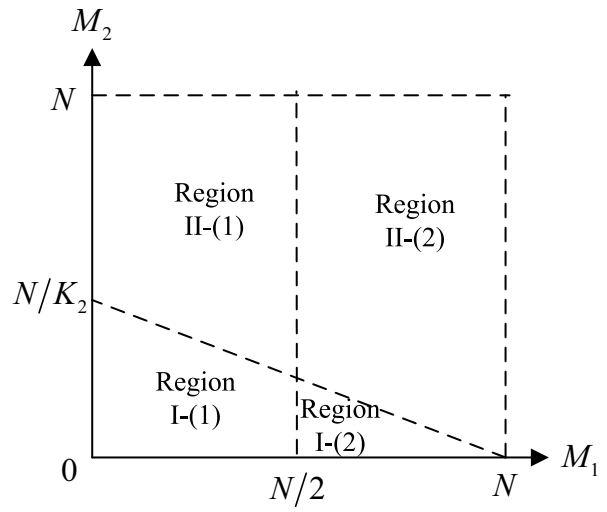

Fig. 2. Different regions of $\left(M_{1}, M_{2}\right)$.

as the achievable rate region of the hybrid caching scheme, where $\mathbb{R}_{+}^{2}$ denotes the positive quadrant and the addition corresponds to the Minkowski sum between sets.

Definition 2: For normalized memory size $M_{1}, M_{2} \geq 0$, we define

$$
\mathcal{R}\left(M_{1}, M_{2}\right)=\left\{R_{1}^{l b}\left(M_{1}, M_{2}\right), R_{2}^{l b}\left(M_{1}, M_{2}\right)\right\}+\mathbb{R}_{+}^{2}
$$

as the information-theoretic (optimal) rate region, where $R_{1}^{l b}\left(M_{1}, M_{2}\right)$ and $R_{2}^{l b}\left(M_{1}, M_{2}\right)$ are the information-theoretic lower bounds of transmission rates in two layers, respectively.

Due to the complicated expressions of the transmission rates in two layers, it is difficult to obtain the optimal $(\alpha, \beta)$ and minimize the transmission rates in two layers simultaneously. Instead, we adopt a heuristic algorithm to optimize $(\alpha, \beta)$ with the following four steps.

1) We observe that the aggregated storage size of each subnetwork (including the storage size of each helper and the storage size of each attached user) is crucial to the transmission rate/traffic load in the considered network. Then, we divide the feasible region of $\left(M_{1}, M_{2}\right)$ into two regions: Region I $\left(M_{1}+K_{2} M_{2}<N\right)$ and Region II $\left(M_{1}+K_{2} M_{2} \geq N\right)$. In particular, $M_{1}+K_{2} M_{2}<N$ refers to the region in which the aggregated storage size of each sub-network is smaller than the overall size of the files in the server, and $M_{1}+K_{2} M_{2} \geq N$ refers to the region in which the aggregated storage size of each sub-network is no smaller than the overall size of the files in the server.

2) We select multiple tuples of $(\alpha, \beta)$ with typical values in region I $\left(M_{1}+K_{2} M_{2}<N\right)$ and evaluate the corresponding transmission rates via (10) and (11). The detailed selection of multiple tuples of $(\alpha, \beta)$ is as follows: In Region I, the aggregated storage size of each sub-network is smaller than the overall size of the files in the server. Then, we need to carefully design $(\alpha, \beta)$ and make use of the small caches at helpers/users. In particular, there may exist three kinds of multi-cast opportunities in the considered network. The first one is the multi-cast opportunity from the server to the helpers (namely, MO-1). The second one is the multicast opportunity from the server to the users (namely, MO-2). The third one is the multi-cast opportunity from each helper to its attached users (namely, MO3). Ideally, we need to exploit the three kinds of multicast opportunities simultaneously to minimize the traffic load in the network. However, it is challenging to conduct the theoretical analysis when exploiting the three kinds of multi-cast opportunities simultaneously. Instead, we choose typical tuples of $(\alpha, \beta)$ such that two kinds of multi-cast opportunities can be exploited simultaneously. According to [10], $(\alpha, \beta)=\left(\frac{M_{1}}{N}, \frac{M_{1}}{N}\right)$ is able to exploit MO-2 and MO-3 simultaneously, and $(\alpha, \beta)=\left(\frac{M_{1}}{M_{1}+K_{2} M_{2}}, 0\right)$ is able to exploit MO-1 and MO-2 simultaneously. Besides, it is easy to verify that $(\alpha, \beta)=(1,1)$ can exploit MO-1 and MO-3 simultaneously. Therefore, to make use of the small caches at helpers/users in a flexible manner, we choose three tuples of $(\alpha, \beta)$ for Region I as

$$
(\alpha, \beta)=\left\{\begin{array}{l}
\left(\frac{M_{1}}{N}, \frac{M_{1}}{N}\right), \text { Tuple I, } \\
\left(\frac{M_{1}}{M_{1}+K_{2} M_{2}}, 0\right), \text { Tuple II, } \\
(1,1), \text { Tuple III. }
\end{array}\right.
$$

3) We select multiple tuples of $(\alpha, \beta)$ with typical values in region II $\left(M_{1}+K_{2} M_{2} \geq N\right)$ and evaluate the corresponding transmission rates via (10) and (11). The detailed selection of multiple tuples of $(\alpha, \beta)$ is as follows: In Region II, the aggregated storage size of each sub-network is no smaller than the overall size of the files in the server. According to [10], for a small value of $\frac{M_{1}}{N},(\alpha, \beta)=\left(\frac{M_{1}}{N}, \frac{M_{1}}{N}\right)$ is able to balance the traffic loads (i.e., $R_{1}^{H}(\alpha, \beta)$ and $R_{2}^{H}(\alpha, \beta)$ ) in the two layers. Then, we choose $(\alpha, \beta)=\left(\frac{M_{1}}{N}, \frac{M_{1}}{N}\right)$ as a candidate tuple of $(\alpha, \beta)$ for a small $\frac{M_{1}}{N}$. For a relatively large value of $\frac{M_{1}}{N}$, if we still choose $(\alpha, \beta)=\left(\frac{M_{1}}{N}, \frac{M_{1}}{N}\right)$, the traffic load (i.e., $R_{1}^{H}(\alpha, \beta)$ ) from the server to helpers may be unacceptably large since $R_{1}^{H}(\alpha, \beta)$ increases as $\beta$ grows. Thus, for a relatively large value of $\frac{M_{1}}{N}$, we choose $(\alpha, \beta)=\left(\frac{M_{1}}{N}, \frac{1}{2}\right)$ to balance the traffic loads in the two layers. To summarize, we choose two tuples of $(\alpha, \beta)$ for Region II as

$$
(\alpha, \beta)=\left\{\begin{array}{l}
\left(\frac{M_{1}}{N}, \frac{M_{1}}{N}\right), \text { Tuple I, } \\
\left(\frac{M_{1}}{N}, \frac{1}{2}\right), \text { Tuple II. }
\end{array}\right.
$$

4) We observe that the gap between the achievable rate region $\mathcal{R}^{H}\left(M_{1}, M_{2}\right)$ and the information-theoretic (optimal) rate region $\mathcal{R}\left(M_{1}, M_{2}\right)$ is dominated by the difference between the achievable rate and its informationtheoretic lower bound in the first layer from the server to the helpers. To reduce the gap between the achievable rate region $\mathcal{R}^{H}\left(M_{1}, M_{2}\right)$ and the informationtheoretic (optimal) rate region $\mathcal{R}\left(M_{1}, M_{2}\right)$, we calculate the transmission rates of the two layers with the three tuples of $(\alpha, \beta)$ in (14) and choose the tuple that minimizes $R_{1}^{H}(\alpha, \beta)$ as an optimized design of $(\alpha, \beta)$, i.e., $\left(\alpha^{*}, \beta^{*}\right)$, for Region I. Similarly, we calculate the 
corresponding transmission rates of the two layers with the two tuples in (15) of $(\alpha, \beta)$ and choose the tuple that minimizes $R_{1}^{H}(\alpha, \beta)$ as an optimized design of $(\alpha, \beta)$, i.e., $\left(\alpha^{*}, \beta^{*}\right)$, for Region II.

To this end, we obtain an optimized $\left(\alpha^{*}, \beta^{*}\right)$, and the corresponding transmission rates $R_{1}^{H}\left(\alpha^{*}, \beta^{*}\right)$ and $R_{2}^{H}\left(\alpha^{*}, \beta^{*}\right)$ in each region. With the optimized $\left(\alpha^{*}, \beta^{*}\right)$, we can characterize the gap between the achievable rate region of the proposed hybrid caching scheme and the information-theoretic rate region in the following theorem.

Theorem 1: For $M_{1} \leq N$ and $M_{2} \leq N$, we have

$$
\mathcal{R}^{H}\left(M_{1}, M_{2}\right) \subset \mathcal{R}\left(M_{1}, M_{2}\right) \subset \frac{1}{48} \mathcal{R}^{H}\left(M_{1}, M_{2}\right)-4 .
$$

Sketches of proof: Since it is straightforward to obtain $\mathcal{R}^{H}\left(M_{1}, M_{2}\right) \subset \mathcal{R}\left(M_{1}, M_{2}\right)$, we only prove $\mathcal{R}\left(M_{1}, M_{2}\right) \subset$ $\frac{1}{48} \mathcal{R}^{H}\left(M_{1}, M_{2}\right)-4$.

Firstly, we let $R_{1}^{l b}\left(M_{1}, M_{2}\right)$ and $R_{2}^{l b}\left(M_{2}\right)$ represent the information-theoretic lower bounds of transmission rates in two layers, respectively. Based on the cut-set theorem [37], we have [14]:

$$
R_{1}^{l b}\left(M_{1}, M_{2}\right) \triangleq \max _{\substack{s_{1} \in\left\{1,2, \cdots, K_{1}\right\} \\ s_{2} \in\left\{1,2, \cdots, K_{2}\right\}}} \frac{s_{1} s_{2}\left(N-s_{1} M_{1}-s_{1} s_{2} M_{2}\right)}{N+s_{1} s_{2}}
$$

and

$$
R_{2}^{l b}\left(M_{2}\right) \triangleq \max _{t \in\left\{1,2, \cdots, K_{2}\right\}} \frac{t\left(N-t M_{2}\right)}{N+t} .
$$

Secondly, we denote the upper bounds of transmission rates $R_{1}^{H}\left(\alpha^{*}, \beta^{*}\right)$ and $R_{2}^{H}\left(\alpha^{*}, \beta^{*}\right)$ as $R_{1}^{u b}\left(M_{1}, M_{2}\right)$ and $R_{2}^{u b}\left(M_{2}\right)$, respectively. Then, we calculate the upper bounds in Appendix A and prove that $R_{1}^{l b}\left(M_{1}, M_{2}\right) \geq \frac{1}{48} R_{1}^{u b}\left(M_{1}, M_{2}\right)-4$ and $R_{2}^{l b}\left(M_{2}\right) \geq \frac{1}{48} R_{2}^{u b}\left(M_{2}\right)-4$ can be satisfied simultaneously in Appendix B and Appendix C, respectively. More specifically, we divide Region I and Region II into two sub-regions based on the value of $M_{1}$, i.e., whether $M_{1}$ is larger or smaller than $N / 2$. Therefore, we have four sub-regions in total to investigate, i.e.,

$$
\begin{cases}M_{1}+K_{2} M_{2}<N \text { and } M_{1}<N / 2, & \text { Sub-region I-(1), } \\ M_{1}+K_{2} M_{2}<N \text { and } M_{1} \geq N / 2, & \text { Sub-region I-(2), } \\ M_{1}+K_{2} M_{2} \geq N \text { and } M_{1}<N / 2, & \text { Sub-region II-(1), } \\ M_{1}+K_{2} M_{2} \geq N \text { and } M_{1} \geq N / 2, & \text { Sub-region II-(2). }\end{cases}
$$

After characterizing the gap between $R_{1}^{l b}\left(M_{1}, M_{2}\right)$ and $R_{1}^{u b}\left(M_{1}, M_{2}\right)$, and the gap between $R_{2}^{l b}\left(M_{2}\right)$ and $R_{2}^{u b}\left(M_{2}\right)$ in the four regions, we summarize that $R_{1}^{l b}\left(M_{1}, M_{2}\right) \geq$ $\frac{1}{48} R_{1}^{u b}\left(M_{1}, M_{2}\right)-4$ and $R_{2}^{l b}\left(M_{2}\right) \geq \frac{1}{48} R_{2}^{u b}\left(M_{2}\right)-4$ can be achieved simultaneously for all possible $\left(M_{1}, M_{2}\right)$.

Remark 3 (Performance comparison with [14]): Similar to the definition in (13), we define $\mathcal{R}^{G}\left(M_{1}, M_{2}\right)$ as the achievable rate region of the generalized caching scheme in [14]. Accordingly to the results in [14], we have

$$
\mathcal{R}^{G}\left(M_{1}, M_{2}\right) \subset \mathcal{R}\left(M_{1}, M_{2}\right) \subset \frac{1}{60} \mathcal{R}^{G}\left(M_{1}, M_{2}\right)-16 .
$$

By comparing the multiplicative and additive factors in 16 and (20), the achievable rate region of the proposed hybrid caching scheme is better than that of the generalized caching scheme in [14]. The improvement results from three aspects. The first aspect is the utilization of the CCG, which leads to smaller achievable rates. The second aspect is the optimization of $\alpha$ and $\beta$. The third aspect is a better quantification of the information-theoretic gap, including a better division of the entire region in Fig. 2 and an improved mathematical proof. It should be pointed out that the improvement of the multiplicative and additive factors is obtained by exploiting the gains in the three aspects simultaneously, and any absence of the gains in the three aspects may fail to achieve the improvement. For instance, we would not be able to obtain the same improvement with the division of the subregions in [10], even if we exploit the former two gains.

Remark 4: (Optimized division of the entire region in Fig. 2 compared with [14]): Basically, the division of the entire region in Fig. 2 is optimized based on the following two observations. On one hand, we observe that the multiplicative factor is mainly determined by the gap between the achievable rate region and the information-theoretic (optimal) rate region in Region I. To optimize the multiplicative factor, we further divide Region I into two sub-regions, i.e., Region I-(1) and Region I-(2). On the other hand, we observe that the additive factor is mainly determined by the gap between the achievable rate region and the information-theoretic (optimal) rate region in Region II. To obtain a better additive factor, we optimize the division of Region II compared with [10]. It is worth pointing out that, dividing the entire region in Fig. 2 into more sub-regions may further improve the quantifications of the multiplicative and additive factors. Nevertheless, this requires a more complicated analysis and will be one of our future works.

\section{Simulation RESUlTS}

In this section, we provide simulation results to show the advantage of the proposed caching scheme over the caching scheme in [10]. In particular, we adopt the overall traffic load in the network, i.e., $R_{1}+K_{1} R_{2}$, as the metric. Next, we first show the traffic load comparison with general values of $\alpha$ and $\beta$, and then give the traffic load comparison with optimized $\alpha$ and $\beta$ in (14) and (15).

Fig. 3 and Fig. 4 illustrate the traffic load comparison with general values of $\alpha$ and $\beta$ in Region I $\left(M_{1}+K_{2} M_{2}<N\right)$ and Region II $\left(M_{1}+K_{2} M_{2} \geq N\right)$, respectively. In particular, we consider the network setting in Region I as follows: $F=1$ M bits, $N=100, K_{1}=10, K_{2}=5, M_{1}=50$, and $M_{2}=8$. Similarly, we consider the network setting in Region II as follows: $F=1 \mathrm{M}$ bits, $N=100, K_{1}=10, K_{2}=5, M_{1}=$ 50 , and $M_{2}=30$. Both figures show that the proposed caching scheme achieves a traffic load similar to the caching scheme in [14] for a small $\alpha$, and the proposed caching scheme is able to effectively reduce the traffic load compared with the caching scheme in [14] for a relatively large $\alpha$. Besides, the traffic load gap of the two caching schemes becomes large as $\alpha$ increases. The reason is as follows: On one hand, a small $\alpha$ leads to a low CCG of the proposed caching scheme (this can be easily verified from (10)), which has little impact on the traffic load reduction compared with the caching scheme in [14]; On the 


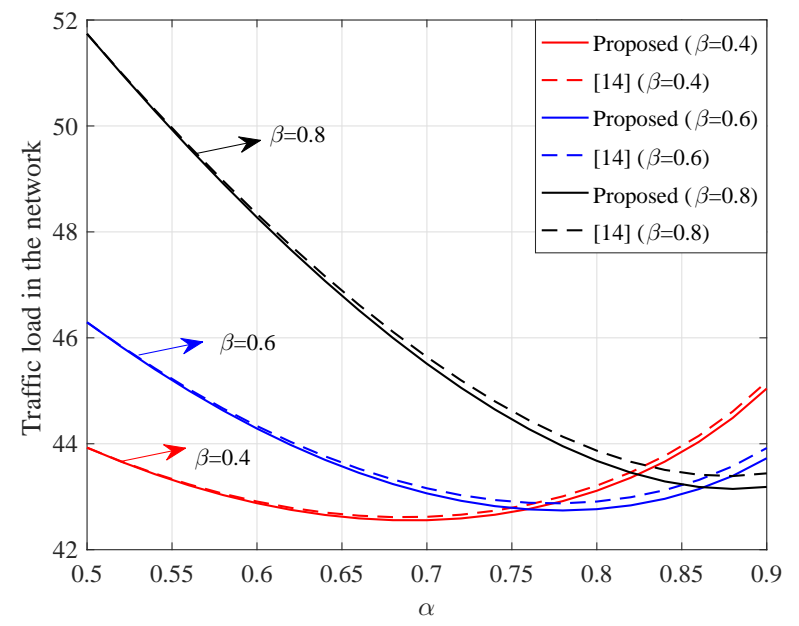

Fig. 3. Comparison of the traffic load in the network in Region I with different $\alpha$ and $\beta$.

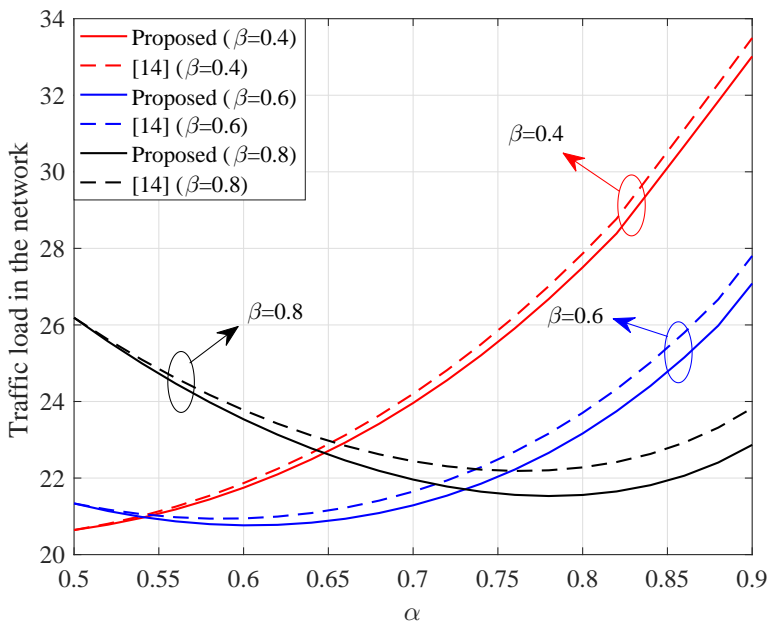

Fig. 4. Comparison of the traffic load in the network in Region II with different $\alpha$ and $\beta$.

other hand, a large $\alpha$ leads to a high CCG, which contributes to a significant traffic load reduction compared with the caching scheme in [14]. Furthermore, by comparing the two figures, we observe that the proposed caching scheme is able to provide larger reductions of traffic load in Region II than in Region I. This is because, the traffic load reduction is proportional to $M_{2}$, which is small in Region I and is relatively large in Region II.

Fig. 5 compares the traffic load in the network between the proposed caching scheme and the caching scheme in [14] as a function of $M_{2}$. In particular, we consider the network setting as follows: $F=1 \mathrm{M}$ bits, $N=100, K_{1}=10$, and $K_{2}=5$. In addition, the traffic load of the proposed algorithm is evaluated with the optimized $\alpha$ and $\beta$ in (14) and (15). The traffic load of the algorithm in [14] is evaluated with its optimized $\alpha$ and $\beta$. From the figure, we observe that, the proposed caching scheme achieves a traffic load similar to the caching scheme in [14] in Region I, i.e., $M_{2}$ is small and satisfies $M_{1}+K_{2} M_{2}<N$. Meanwhile, the proposed caching scheme is able to effectively

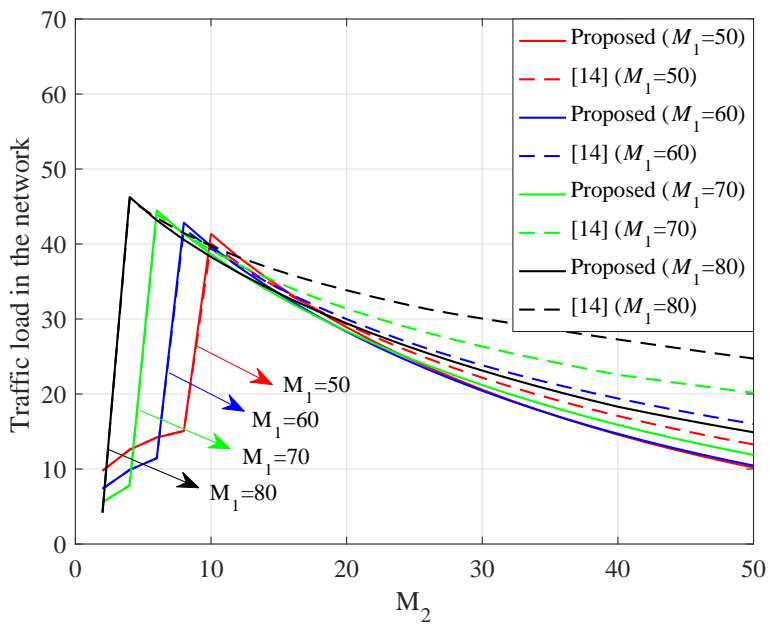

Fig. 5. Comparison of the traffic load in the network with optimized $\alpha$ and $\beta$ in (14) and (15).

reduce the traffic load compared with the caching scheme in [14] in Region II, i.e., $M_{2}$ is relatively large and satisfies $M_{1}+K_{2} M_{2} \geq N$. Furthermore, the traffic load gap of the two caching schemes becomes large as $M_{2}$ increases. This demonstrates the advantage of the proposed caching scheme compared with the caching scheme in [14].

\section{CONCLUSIONS}

In this paper, we studied the decentralized caching in twolayer networks, in which users request contents from a server and the server satisfies users through helpers. By simultaneously utilizing the CCG, SMO, and CMO, we developed an hybrid caching scheme to reduce the traffic loads/transmission rates from both the server and the helpers. Besides, we analytically derived the transmission rates and demonstrated that the hybrid caching scheme is able to reduce the transmission rate from the server without increasing the transmission rate from each helper compared with the caching scheme in [14]. Furthermore, we theoretically analyzed the performance limit of the proposed caching scheme and demonstrated that the achievable rate region of the proposed caching scheme lies within constant margins to the information-theoretic optimum. In particular, the multiplicative and additive factors are carefully sharpened to be $\frac{1}{48}$ and 4 respectively, both of which are better than those in [14]. Finally, simulation results demonstrated the advantage of the proposed caching scheme compared with the caching scheme in [14] in terms of the overall traffic load in the network.

\section{APPENDIX A}

Upper Bounds $R_{1}^{u b}\left(M_{1}, M_{2}\right)$ AND $R_{2}^{u b}\left(M_{2}\right)$

Since the transmission rates $\left(R_{1}^{H}(\alpha, \beta), R_{2}^{H}(\alpha, \beta)\right)$ are highly related to the values of $(\alpha, \beta)$, the upper bounds $R_{1}^{u b}\left(M_{1}, M_{2}\right)$ and $R_{2}^{u b}\left(M_{2}\right)$ are also determined by $(\alpha, \beta)$. Meanwhile, transmission rates differ in variable $\left(M_{1}, M_{2}\right)$ regimes. Thus, we first consider two regimes, i.e., Regime I) $M_{1}+K_{2} M_{2} \leq N$ and Regime II) $M_{1}+K_{2} M_{2}>N$. In what 
follows, we will discuss the upper bounds $R_{1}^{u b}\left(M_{1}, M_{2}\right)$ and $R_{2}^{u b}\left(M_{2}\right)$ in the two regimes, respectively.

\section{A. Upper Bounds $R_{1}^{u b}\left(M_{1}, M_{2}\right)$ and $R_{2}^{u b}\left(M_{2}\right)$ in Regime I}

In this regime, we consider three tuples of $(\alpha, \beta)$. Tuple I is $(\alpha, \beta)=\left(\frac{M_{1}}{N}, \frac{M_{1}}{N}\right)$, Tuple II is $(\alpha, \beta)=\left(\frac{M_{1}}{M_{1}+K_{2} M_{2}}, 0\right)$, Tuple III is $(\alpha, \beta)=(1,1)$.

Firstly, we substitute $(\alpha, \beta)=\left(\frac{M_{1}}{N}, \frac{M_{1}}{N}\right)$ into (10) and (11). Then, we have

$$
\begin{aligned}
R_{1}^{H}(\alpha, \beta)= & \left(1-\frac{M_{1}}{N}\right) K_{1} K_{2}\left(1-\frac{M_{2}}{N}\right) \frac{N}{K_{1} K_{2} M_{2}} \times \\
& \left(1-\left(1-\frac{M_{2}}{N}\right)^{K_{1} K_{2}}\right) \leq \frac{N}{M_{2}}\left(1-\frac{M_{2}}{N}\right) \\
\leq & \min \left\{K_{1} K_{2}, \frac{N}{M_{2}}\left(1-\frac{M_{2}}{N}\right)\right\}
\end{aligned}
$$

and

$$
\begin{aligned}
R_{2}^{H}(\alpha, \beta) & =K_{2}\left(1-\frac{M_{2}}{N}\right) \frac{N}{K_{2} M_{2}}\left(1-\left(1-\frac{M_{2}}{N}\right)^{K_{2}}\right) \\
& \leq \min \left\{K_{2}, \frac{N}{M_{2}}\right\} .
\end{aligned}
$$
have

Substitute $(\alpha, \beta)=\left(\frac{M_{1}}{M_{1}+K_{2} M_{2}}, 0\right)$ into (10) and (11), we

$$
\begin{aligned}
R_{1}^{H}(\alpha, \beta) \leq & \frac{M_{1} K_{2}}{M_{1}+K_{2} M_{2}} \min \left\{K_{1}, \frac{N}{M_{1}+M_{2} K_{2}}\right\}+ \\
& \frac{K_{2} M_{2}}{M_{1}+K_{2} M_{2}} \min \left\{K_{1} K_{2}, \frac{N K_{2}}{M_{1}+M_{2} K_{2}}\right\} \\
= & \frac{M_{1}}{M_{1}+K_{2} M_{2}} \min \left\{K_{1} K_{2}, \frac{N K_{2}}{M_{1}+M_{2} K_{2}}\right\}+ \\
& \frac{K_{2} M_{2}}{M_{1}+K_{2} M_{2}} \min \left\{K_{1} K_{2}, \frac{N K_{2}}{M_{1}+M_{2} K_{2}}\right\} \\
\leq & \min \left\{K_{1} K_{2}, \frac{N K_{2}}{M_{1}+M_{2} K_{2}}\right\}
\end{aligned}
$$

and

$$
R_{2}^{H}(\alpha, \beta) \leq K_{2} \stackrel{(a)}{=} \min \left\{K_{2}, \frac{N}{M_{2}}\right\},
$$

where $(a)$ follows from $K_{2} M_{2}<N$ in regime I.

Substitute $(\alpha, \beta)=(1,1)$ into (10) and (11). Then, we have

$$
\begin{aligned}
R_{1}^{H}(\alpha, \beta)= & K_{1} K_{2}\left(1-\frac{M_{1}}{N}\right)\left(1-\frac{M_{2}}{N}\right) \frac{N}{K_{1} M_{1}} \times \\
& \left(1-\left(1-\frac{M_{1}}{N}\right)^{K_{1}}\right)=\frac{K_{2} N}{M_{1}}\left(1-\frac{M_{1}}{N}\right)
\end{aligned}
$$

and

$$
R_{2}^{H}(\alpha, \beta) \leq K_{2} \stackrel{(a)}{=} \min \left\{K_{2}, \frac{N}{M_{2}}\right\},
$$

where $(a)$ follows from $K_{2} M_{2}<N$ in regime I.

Thus, if we choose $\left(\alpha^{*}, \beta^{*}\right)$ in three considered tuples corresponding to the minimum $R_{1}^{H}(\alpha, \beta)$, we can achieve the upper bounds $R_{1}^{u b}\left(M_{1}, M_{2}\right)$ and $R_{2}^{u b}\left(M_{2}\right)$ in Regime I as

$$
\begin{aligned}
R_{1}^{u b}\left(M_{1}, M_{2}\right) \leq & \min \left\{K_{1} K_{2}, \frac{N}{M_{2}}\left(1-\frac{M_{2}}{N}\right), \frac{N K_{2}}{M_{1}+M_{2} K_{2}},\right. \\
& \left.\frac{K_{2} N}{M_{1}}\left(1-\frac{M_{1}}{N}\right)\right\} .
\end{aligned}
$$

and

$$
R_{2}^{u b}\left(M_{2}\right) \leq \min \left\{K_{2}, \frac{N}{M_{2}}\right\}
$$

\section{B. Upper Bounds $R_{1}^{u b}\left(M_{1}, M_{2}\right)$ and $R_{2}^{u b}\left(M_{2}\right)$ in Regime II}

In this regime, we consider the tuples of $(\alpha, \beta)$ as follows: Tuple I is $(\alpha, \beta)=\left(\frac{M_{1}}{N}, \frac{M_{1}}{N}\right)$, and Tuple II is $(\alpha, \beta)=$ $\left(\frac{M_{1}}{N}, \frac{1}{2}\right)$. Since we have obtained the upper bounds with Tuple I in (21) and (22), we will only calculate the upper bounds with Tuple II in the following.

Substitute $(\alpha, \beta)=\left(\frac{M_{1}}{N}, \frac{1}{2}\right)$ into (10) and (11), we have

$$
\begin{aligned}
R_{1}^{H}(\alpha, \beta)= & \left(1-\frac{M_{1}}{N}\right)\left(1-\frac{M_{2}}{2\left(N-M_{1}\right)}\right) \frac{2\left(N-M_{1}\right)}{M_{2}} \times \\
& \left(1-\left(1-\frac{M_{2}}{2\left(N-M_{1}\right)}\right)^{K_{1} K_{2}}\right) \\
\leq & \left(1-\frac{M_{1}}{N}\right) \min \left\{K_{1} K_{2}, \frac{2\left(N-M_{1}\right)}{M_{2}}\right\} \\
\leq & \min \left\{K_{1} K_{2}, \frac{2\left(N-M_{1}\right)^{2}}{N M_{2}}\right\}
\end{aligned}
$$

and

$$
\begin{aligned}
& R_{2}^{H}(\alpha, \beta) \\
= & \frac{M_{1}}{N}\left(1-\frac{M_{2}}{2 M_{1}}\right) \frac{2 M_{1}}{M_{2}}\left(1-\left(1-\frac{M_{2}}{2 M_{1}}\right)^{K_{2}}\right) \\
& +\left(1-\frac{M_{1}}{N}\right)\left(1-\frac{M_{2}}{2\left(N-M_{1}\right)}\right) \frac{2\left(N-M_{1}\right)}{M_{2}} \times \\
& \left(1-\left(1-\frac{M_{2}}{2\left(N-M_{1}\right)}\right)^{K_{2}}\right) \\
\leq & \frac{M_{1}}{N} \min \left\{K_{2}, \frac{2 N}{M_{2}}\right\}+\left(1-\frac{M_{1}}{N}\right) \min \left\{K_{2}, \frac{2 N}{M_{2}}\right\} \\
= & \min \left\{K_{2}, \frac{2 N}{M_{2}}\right\} \leq 2 \min \left\{K_{2}, \frac{N}{M_{2}}\right\} .
\end{aligned}
$$

Thus, if we choose $\left(\alpha^{*}, \beta^{*}\right)$ in two considered tuples corresponding to the minimum $R_{1}^{H}(\alpha, \beta)$, we can achieve the upper bounds $R_{1}^{u b}\left(M_{1}, M_{2}\right)$ and $R_{2}^{u b}\left(M_{2}\right)$ in Regime II as

$R_{1}^{u b}\left(M_{1}, M_{2}\right) \leq \min \left\{K_{1} K_{2}, \frac{N}{M_{2}}\left(1-\frac{M_{2}}{N}\right), \frac{2\left(N-M_{1}\right)^{2}}{N M_{2}}\right\}$

and

$$
R_{2}^{u b}\left(M_{2}\right) \leq 2 \min \left\{K_{2}, \frac{N}{M_{2}}\right\}
$$

respectively. 


\section{APPENDIX B}

GAP BETWEEN THE UPPER BOUND $R_{1}^{u b}\left(M_{1}, M_{2}\right)$ AND LOWER BOUND $R_{1}^{l b}\left(M_{1}, M_{2}\right)$

In this section, we will characterize the gap between the upper bound $R_{1}^{u b}\left(M_{1}, M_{2}\right)$ and the lower bound $R_{1}^{l b}\left(M_{1}, M_{2}\right)$. Recall that we consider $K_{1} \geq 2, K_{2} \geq 2$, and $N \geq K_{1} K_{2}$.

\section{A. Gap between $R_{1}^{u b}\left(M_{1}, M_{2}\right)$ and $R_{1}^{l b}\left(M_{1}, M_{2}\right)$ in Regime} I

In regime I, we consider two subregimes, i.e., Subregime I) $0 \leq M_{1} \leq \frac{N}{2}$ and Subregime II) $\frac{N}{2} \leq M_{1} \leq N$. Then, we will discuss the gap in the two subregimes respectively.

1) Gap in Subregime I: In this subregime, we have $M_{1}+$ $K_{2} M_{2} \leq N$ and $0 \leq M_{1} \leq \frac{N}{2}$. Then, we have $0 \leq M_{2} \leq$ $\frac{N-M_{1}}{K_{2}} \leq \frac{N}{2}$ using $K_{2} \geq 2$. Thus, we consider:

A) $0 \leq M_{1}<\frac{N}{2 K_{1}}$ and $0 \leq M_{2} \leq \frac{N}{K_{1} K_{2}}$

B) $0 \leq M_{1}<\frac{N}{2 K_{1}}$ and $\frac{N}{K_{1} K_{2}} \leq M_{2} \leq \frac{N}{2 K_{2}}$;

C) $0 \leq M_{1}<\frac{N}{2 K_{1}}$ and $\frac{N}{2 K_{2}} \leq M_{2} \leq \frac{N}{2}$;

D) $\frac{N}{2 K_{1}} \leq M_{1}<\frac{N}{4}$ and $0 \leq M_{2} \leq \frac{N}{4 K_{2}}$;

E) $\frac{N}{2 K_{1}} \leq M_{1}<\frac{N}{4}$ and $\frac{N}{4 K_{2}} \leq M_{2} \leq \frac{N}{2}$;

F) $\frac{N}{4} \leq M_{1}<\frac{N}{2}$ and $0 \leq M_{2} \leq \frac{N-M_{1}}{2 K_{2}}$;

G) $\frac{N}{4} \leq M_{1}<\frac{N}{2}$ and $\frac{N-M_{1}}{2 K_{2}} \leq M_{2} \leq \frac{N-M_{1}}{K_{2}}$.

- A) $0 \leq M_{1}<\frac{N}{2 K_{1}}$ and $0 \leq M_{2} \leq \frac{N}{K_{1} K_{2}}$ : we choose $s_{1}=\left\lfloor\frac{K_{1}}{2}\right\rfloor$ and $s_{2}=K_{2}$ in the lower bound (17). This is valid choice since $K_{1} \geq 2$, and thus $s_{1}=\left\lfloor\frac{K_{1}}{2}\right\rfloor \geq 1$. Then, we have

$$
\begin{aligned}
R_{1}^{l b}\left(M_{1}, M_{2}\right) & \geq \frac{\left\lfloor\frac{K_{1}}{2}\right\rfloor K_{2}\left(N-\left\lfloor\frac{K_{1}}{2}\right\rfloor M_{1}-\left\lfloor\frac{K_{1}}{2}\right\rfloor K_{2} M_{2}\right)}{N+\left\lfloor\frac{K_{1}}{2}\right\rfloor K_{2}} \\
& \stackrel{(a)}{\geq} \frac{\frac{K_{1} K_{2}}{4}\left(N-\frac{M_{1} K_{1}}{2}-\frac{M_{2} K_{1} K_{2}}{2}\right)}{N+\frac{K_{1} K_{2}}{2}} \\
& \stackrel{(b)}{\geq} \frac{\frac{K_{1} K_{2}}{4}\left(N-\frac{N}{4}-\frac{N}{2}\right)}{N+\frac{N}{2}} \\
& =\frac{K_{1} K_{2}}{24}
\end{aligned}
$$

where (a) follows from $x \geq\lfloor x\rfloor \geq \frac{x}{2}$ for any $x \geq 1$ and $(b)$ follows from $\frac{K_{1} M_{1}}{2} \leq \frac{N}{4}$ by using $M_{1} \leq \frac{N}{2 K_{1}}, \frac{K_{1} K_{2} M_{2}}{2} \leq \frac{N}{2}$ by using $M_{2} \leq \frac{N}{K_{1} K_{2}}, \frac{K_{1} K_{2}}{2} \leq \frac{N}{2}$ by using $N \geq K_{1} K_{2}$. Combining with (27), we have

$$
\begin{aligned}
& R_{1}^{l b}\left(M_{1}, M_{2}\right) \geq \frac{K_{1} K_{2}}{24} \geq \frac{1}{24} \min \left\{K_{1} K_{2}, \frac{N}{M_{2}}\left(1-\frac{M_{2}}{N}\right),\right. \\
& \left.\frac{N K_{2}}{M_{1}+M_{2} K_{2}}, \frac{K_{2} N}{M_{1}}\left(1-\frac{M_{1}}{N}\right)\right\} \geq \frac{1}{24} R_{1}^{u b}\left(M_{1}, M_{2}\right) .
\end{aligned}
$$

- B) $0 \leq M_{1}<\frac{N}{2 K_{1}}$ and $\frac{N}{K_{1} K_{2}} \leq M_{2} \leq \frac{N}{2 K_{2}}$ : we choose $s_{1}=\left|\frac{N}{2 M_{2} K_{2}}\right|$ and $s_{2}=K_{2}$ in the lower bound (17). Note that this is a valid choice since

$$
1 \stackrel{(a)}{\leq}\left\lfloor\frac{N}{2 M_{2} K_{2}}\right\rfloor \stackrel{(b)}{\leq} \frac{N}{2 M_{2} K_{2}} \stackrel{(c)}{\leq} \frac{K_{1}}{2},
$$

where $(a)$ follows from $M_{2} \leq \frac{N}{2 K_{2}},(b)$ follows from $x \geq\lfloor x\rfloor$ for any $x \geq 1$, and $(c)$ follows from $\frac{N}{K_{1} K_{2}} \leq M_{2}$.
Substitute $s_{1}$ and $s_{2}$ into the lower bound (17), we have

$$
\begin{aligned}
& R_{1}^{l b}\left(M_{1}, M_{2}\right) \\
& \geq \frac{\left\lfloor\frac{N}{2 M_{2} K_{2}}\right\rfloor K_{2}\left(N-\left\lfloor\frac{N}{2 M_{2} K_{2}}\right\rfloor M_{1}-\left\lfloor\frac{N}{2 M_{2} K_{2}}\right\rfloor K_{2} M_{2}\right)}{N+\left\lfloor\frac{N}{2 M_{2} K_{2}}\right\rfloor K_{2}} \\
& \text { (a) } \frac{\frac{N}{4 M_{2}}\left(N-\frac{N M_{1}}{2 M_{2} K_{2}}-\frac{N}{2}\right)}{N+\frac{N}{2 M_{2}}}=\frac{\frac{N}{4 M_{2}}\left(\frac{1}{2}-\frac{M_{1}}{2 M_{2} K_{2}}\right)}{1+\frac{1}{2 M_{2}}} \\
& \stackrel{\text { (b) } \frac{N}{4 M_{2}}\left(\frac{1}{2}-\frac{1}{4}\right)}{1+\frac{1}{2}}=\frac{N}{24 M_{2}} \geq \frac{N}{24 M_{2}}\left(1-\frac{M_{2}}{N}\right) \text {, }
\end{aligned}
$$

where (a) follows from $x \geq\lfloor x\rfloor \geq \frac{x}{2}$ for any $x \geq 1$, (b) follows from $\frac{M_{1}}{2 M_{2} K_{2}} \leq \frac{N /\left(2 K_{1}\right)}{2 N / K_{1}} \leq \frac{1}{4}$ since $M_{1}<\frac{N}{2 K_{1}}$ and $M_{2} \geq \frac{N}{K_{1} K_{2}}$, and $\frac{1}{2 M_{2}} \leq \frac{K_{1} K_{2}}{2 N} \leq \frac{1}{2}$ using $\frac{N}{K_{1} K_{2}} \leq M_{2}$ and $N \geq K_{1} K_{2}$. Combining with (27), we have

$$
\begin{aligned}
R_{1}^{l b}\left(M_{1}, M_{2}\right) \geq & \frac{N}{24 M_{2}}\left(1-\frac{M_{2}}{N}\right) \\
\geq & \frac{1}{24} \min \left\{K_{1} K_{2}, \frac{N}{M_{2}}\left(1-\frac{M_{2}}{N}\right),\right. \\
& \left.\frac{N K_{2}}{M_{1}+M_{2} K_{2}}, \frac{K_{2} N}{M_{1}}\left(1-\frac{M_{1}}{N}\right)\right\} \\
\geq & \frac{1}{24} R_{1}^{u b}\left(M_{1}, M_{2}\right) .
\end{aligned}
$$

- C) $0 \leq M_{1}<\frac{N}{2 K_{1}}$ and $\frac{N}{2 K_{2}} \leq M_{2} \leq \frac{N}{2}$ : we choose $s_{1}=1$ and $s_{2}=\left\lfloor\frac{N}{2 M_{2}}\right\rfloor$ in the lower bound (17). This is a valid choice since

$$
1 \leq\left\lfloor\frac{N}{2 \cdot N / 2}\right\rfloor \stackrel{(a)}{\leq}\left\lfloor\frac{N}{2 M_{2}}\right\rfloor \stackrel{(b)}{\leq} \frac{N}{2 M_{2}} \stackrel{(c)}{\leq} K_{2}
$$

where (a) follows from $M_{2} \leq \frac{N}{2}$, (b) follows from $x \geq\lfloor x\rfloor$ for any $x \geq 1$, and (c) follows from $\frac{N}{2 K_{2}} \leq M_{2}$.

Substitute $s_{1}$ and $s_{2}$ into the lower bound (17), we have

$$
\begin{aligned}
& R_{1}^{l b}\left(M_{1}, M_{2}\right) \geq \frac{\left\lfloor\frac{N}{2 M_{2}}\right\rfloor\left(N-M_{1}-\left\lfloor\frac{N}{2 M_{2}}\right\rfloor M_{2}\right)}{N+\left\lfloor\frac{N}{2 M_{2}}\right\rfloor} \\
& \text { (a) } \frac{\frac{N}{4 M_{2}}\left(N-M_{1}-\frac{N}{2}\right)}{N+\frac{N}{2 M_{2}}}=\frac{\frac{N}{4 M_{2}}\left(\frac{1}{2}-\frac{M_{1}}{N}\right)}{1+\frac{1}{2 M_{2}}} \\
& \stackrel{\text { (b) }}{\geq} \frac{\frac{N}{4 M_{2}}\left(\frac{1}{2}-\frac{1}{4}\right)}{1+\frac{1}{2}}=\frac{N}{24 M_{2}} \geq \frac{N}{24 M_{2}}\left(1-\frac{M_{2}}{N}\right) \text {, }
\end{aligned}
$$

where (a) follows from $x \geq\lfloor x\rfloor \geq \frac{x}{2}$ for any $x \geq 1$, (b) follows from $\frac{M_{1}}{N} \leq \frac{N /\left(2 K_{1}\right)}{N} \leq \frac{1}{4}$ using $M_{1} \leq \frac{N}{2 K_{1}}$ and $K_{1} \geq$ 2 , and $\frac{1}{2 M_{2}} \leq \frac{K_{2}}{N} \leq \frac{K_{1} K_{2}}{K_{1} N} \leq \frac{1}{K_{1}} \leq \frac{1}{2}$ using $\frac{N}{2 K_{2}} \leq M_{2}$, $N \geq K_{1} K_{2}$, and $K_{1} \geq 2$. Combining with (27), we have

$$
R_{1}^{l b}\left(M_{1}, M_{2}\right) \geq \frac{N}{24 M_{2}}\left(1-\frac{M_{2}}{N}\right) \geq \frac{1}{24} R_{1}^{u b}\left(M_{1}, M_{2}\right) .
$$

- D) $\frac{N}{2 K_{1}} \leq M_{1}<\frac{N}{4}$ and $0 \leq M_{2} \leq \frac{N}{4 K_{2}}$ : we choose $s_{1}=\left\lfloor\frac{N}{2\left(M_{1}+M_{2} K_{2}\right)}\right\rfloor$ and $s_{2}=K_{2}$ in the lower bound (17). 
This is a valid choice since

$$
\begin{aligned}
& 1=\left\lfloor\frac{N}{2(N / 4+N / 4)}\right\rfloor \stackrel{(a)}{\leq}\left\lfloor\frac{N}{2\left(M_{1}+M_{2} K_{2}\right)}\right\rfloor \\
& \stackrel{(b)}{\leq} \frac{N}{2\left(M_{1}+M_{2} K_{2}\right)} \leq \frac{N}{2 M_{1}} \stackrel{(c)}{\leq} K_{1},
\end{aligned}
$$

where ( $a$ ) follows from $M_{1} \leq \frac{N}{4}$ and $M_{2} \leq \frac{N}{4 K_{2}}$, (b) follows from $x \geq\lfloor x\rfloor$ for any $x \geq 1$, and (c) follows from $\frac{N}{2 K_{1}} \leq M_{1}$.

Then, we have

$$
\begin{aligned}
& R_{1}^{l b}\left(M_{1}, M_{2}\right) \\
& \geq \frac{\left\lfloor\frac{N}{2\left(M_{1}+M_{2} K_{2}\right)}\right\rfloor K_{2}\left(N-\left\lfloor\frac{N}{2\left(M_{1}+M_{2} K_{2}\right)}\right\rfloor\left(M_{1}+M_{2} K_{2}\right)\right)}{N+\left\lfloor\frac{N}{2\left(M_{1}+M_{2} K_{2}\right)}\right\rfloor K_{2}} \\
& \stackrel{(a) \frac{N K_{2}}{4\left(M_{1}+M_{2} K_{2}\right)}\left(N-\frac{N}{2\left(M_{1}+M_{2} K_{2}\right)}\left(M_{1}+K_{2} M_{2}\right)\right)}{N+\frac{N K_{2}}{2\left(M_{1}+M_{2} K_{2}\right)}}
\end{aligned}
$$

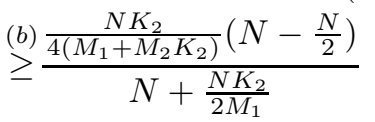

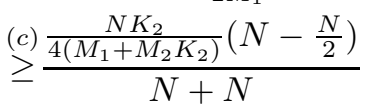

$$
\begin{aligned}
& =\frac{N K_{2}}{16\left(M_{1}+M_{2} K_{2}\right)} \text {, }
\end{aligned}
$$

where $(a)$ follows from $x \geq\lfloor x\rfloor \geq \frac{x}{2}$ for any $x \geq 1$, (b) follows from $\frac{N K_{2}}{2\left(M_{1}+M_{2} k_{2}\right)} \leq \frac{N K_{2}}{2 M_{1}}$, and (c) follows from $\frac{N K_{2}}{2 M_{1}} \leq K_{1} K_{2} \leq N$ by using $\frac{N}{2 K_{1}} \leq M_{1}$. Combining with (27), we have

$$
\begin{aligned}
R_{1}^{l b}\left(M_{1}, M_{2}\right) \geq & \frac{N K_{2}}{16\left(M_{1}+M_{2} K_{2}\right)} \\
\geq & \frac{1}{16} \min \left\{K_{1} K_{2}, \frac{N}{M_{2}}\left(1-\frac{M_{2}}{N}\right),\right. \\
& \left.\frac{N K_{2}}{M_{1}+M_{2} K_{2}}, \frac{K_{2} N}{M_{1}}\left(1-\frac{M_{1}}{N}\right)\right\} \\
\geq & \frac{1}{16} R_{1}^{u b}\left(M_{1}, M_{2}\right) .
\end{aligned}
$$

- E) $\frac{N}{2 K_{1}} \leq M_{1}<\frac{N}{4}$ and $\frac{N}{4 K_{2}} \leq M_{2} \leq \frac{N}{2}$ : Let

$$
\left(s_{1}, s_{2}\right)=\left\{\begin{array}{lr}
\left(\left\lfloor\frac{N}{4 M_{1}}\right\rfloor,\left\lfloor\frac{M_{1}}{M_{2}}\right\rfloor\right), & \text { if } M_{1} \geq M_{2}, \\
\left(\left\lfloor\frac{N}{4 M_{2}}\right\rfloor, 1\right), & \text { otherwise. }
\end{array}\right.
$$

in the lower bound (17). This is a valid choice since for $M_{1} \geq$ $M_{2}$, we have

$$
1=\left\lfloor\frac{N}{4 \cdot N / 4}\right\rfloor \stackrel{(a)}{\leq}\left\lfloor\frac{N}{4 M_{1}}\right\rfloor \stackrel{(b)}{\leq} \frac{N}{4 M_{1}} \stackrel{(c)}{\leq} \frac{K_{1}}{2}
$$

and

$$
1=\left\lfloor\frac{M_{1}}{M_{2}}\right\rfloor \leq \frac{M_{1}}{M_{2}} \stackrel{(d)}{\leq} \frac{N / 4}{N /\left(4 K_{2}\right)}=K_{2},
$$

where (a) follows from $M_{1}<\frac{N}{4}$, (b) follows from $x \geq\lfloor x\rfloor \geq$ $\frac{x}{2}$ for any $x \geq 1,(c)$ follows from $\frac{N}{2 K_{1}} \leq M_{1}$, and $(d)$ follows from $M_{1} \leq \frac{N}{4}$ and $M_{2} \geq \frac{N}{4 K_{2}}$.
For $M_{1}<M_{2}$, we have

$$
1=\left\lfloor\frac{N}{4 \cdot N / 4}\right\rfloor \leq\left\lfloor\frac{N}{4 M_{2}}\right\rfloor \leq\left\lfloor\frac{N}{4 M_{1}}\right\rfloor \leq \frac{N}{4 M_{1}} \leq \frac{K_{1}}{2}
$$

Note that $s_{1} \leq \frac{N}{4 M_{1}}$ and $\frac{N}{16 M_{2}} \leq s_{1} s_{2} \leq \frac{N}{4 M_{2}}$ due to $x \geq$ $\lfloor x\rfloor \geq \frac{x}{2}$ for any $x \geq 1$. Then, we have

$$
\begin{aligned}
& R_{1}^{l b}\left(M_{1}, M_{2}\right) \geq \frac{\frac{N}{16 M_{2}}\left(N-\frac{N}{4 M_{1}} M_{1}-\frac{N}{4 M_{2}} M_{2}\right)}{N+\frac{N}{4 M_{2}}}
\end{aligned}
$$

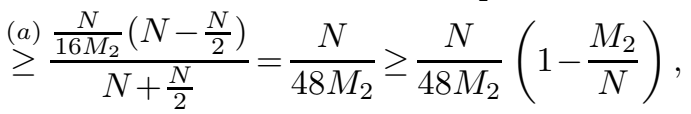

where ( $a$ ) follows from $\frac{N}{4 M_{2}} \leq K_{2}=\frac{K_{1} K_{2}}{K_{1}} \leq \frac{K_{1} K_{2}}{2} \leq \frac{N}{2}$ using $\frac{N}{4 K_{2}} \leq M_{2}, N>K_{1} K_{2}$, and $K_{1} \geq 2$. Combined with (27), we have

$$
R_{1}^{l b}\left(M_{1}, M_{2}\right) \geq \frac{N}{48 M_{2}}\left(1-\frac{M_{2}}{N}\right) \geq \frac{1}{48} R_{1}^{u b}\left(M_{1}, M_{2}\right) .
$$

- F) $\frac{N}{4} \leq M_{1}<\frac{N}{2}$ and $0 \leq M_{2} \leq \frac{N-M_{1}}{2 K_{2}}$ : we choose $s_{1}=1$ and $s_{2}=K_{2}$ in the lower bound (17). Then, we have

$$
\begin{aligned}
R_{1}^{l b}\left(M_{1}, M_{2}\right) & \geq \frac{K_{2}\left(N-M_{1}-M_{2} K_{2}\right)}{N+K_{2}} \\
& \stackrel{(a)}{\geq} \frac{K_{2}\left(N-M_{1}-\frac{N-M_{2}}{2}\right)}{N+N / 2}=\frac{K_{2}\left(N-M_{1}\right)}{3 N} \\
& =\frac{K_{2} N}{3 M_{1}}\left(1-\frac{M_{1}}{N}\right) \cdot \frac{M_{1}}{N} \stackrel{(b)}{\geq} \frac{K_{2} N}{3 M_{1}}\left(1-\frac{M_{1}}{N}\right) \cdot \frac{1}{4} \\
& =\frac{K_{2} N}{12 M_{1}}\left(1-\frac{M_{1}}{N}\right)
\end{aligned}
$$

where ( $a$ ) follows from $x \geq\lfloor x\rfloor \geq \frac{x}{2}$ for any $x \geq 1$ and $K_{2} \leq \frac{K_{1} K_{2}}{K_{1}} \leq \frac{N}{2}$ by using $N \geq K_{1} K_{2}$ and $K_{1} \geq 2$, (b) follows from $\frac{M_{1}}{N} \geq \frac{N / 4}{N}=\frac{1}{4}$. Combining with (27), we have

$$
\begin{aligned}
R_{1}^{l b}\left(M_{1}, M_{2}\right) \geq & \frac{K_{2} N}{12 M_{1}}\left(1-\frac{M_{1}}{N}\right) \geq \frac{1}{12} \min \left\{K_{1} K_{2}\right. \\
& \left.\frac{N}{M_{2}}\left(1-\frac{M_{2}}{N}\right), \frac{N K_{2}}{M_{1}+M_{2} K_{2}}, \frac{K_{2} N}{M_{1}}\left(1-\frac{M_{1}}{N}\right)\right\} \\
\geq & \frac{1}{12} R_{1}^{u b}\left(M_{1}, M_{2}\right) .
\end{aligned}
$$

- G) $\frac{N}{4} \leq M_{1}<\frac{N}{2}$ and $\frac{N-M_{1}}{2 K_{2}} \leq M_{2} \leq \frac{N-M_{1}}{K_{2}}$ : we choose $s_{1}=1$ and $s_{2}=\left\lfloor\frac{N-M_{1}}{2 M_{2}}\right\rfloor$ in the lower bound (17). This is a valid choice since

$$
1 \stackrel{(a)}{\leq}\left\lfloor\frac{N-M_{1}}{K_{2} M_{2}}\right\rfloor \stackrel{(b)}{\leq}\left\lfloor\frac{N-M_{1}}{2 M_{2}}\right\rfloor \stackrel{(c)}{\leq} \frac{N-M_{1}}{2 M_{2}} \stackrel{(d)}{\leq} K_{2}
$$

where ( $a$ ) follows from $M_{1}+K_{2} M_{2} \leq N$, (b) follows from $K_{2} \geq 2$, (c) follows from $x \geq\lfloor x\rfloor$ for any $x \geq 1$, and $(d)$ follows from $\frac{N-M_{1}}{2 K_{2}} \leq M_{2}$. 
Then, we have

$$
\begin{aligned}
& R_{1}^{l b}\left(M_{1}, M_{2}\right) \geq \frac{\left\lfloor\frac{N-M_{1}}{2 M_{2}}\right\rfloor\left(N-M_{1}-\left\lfloor\frac{N-M_{1}}{2 M_{2}}\right\rfloor M_{2}\right)}{N+\left\lfloor\frac{N-M_{1}}{2 M_{2}}\right\rfloor} \\
& \text { (a) } \frac{\frac{N-M_{1}}{4 M_{2}}\left(N-M_{1}-\frac{N-M_{1}}{2 M_{2}} M_{2}\right)}{N+N / 2}=\frac{\left(N-M_{1}\right)^{2}}{12 N M_{2}} \\
& =\frac{K_{2}\left(N-M_{1}\right)}{12 M_{1}} \cdot \frac{N-M_{1}}{M_{2} K_{2}} \cdot \frac{M_{1}}{N} \\
& \stackrel{(b)}{\geq} \frac{K_{2}\left(N-M_{1}\right)}{12 M_{1}} \cdot 1 \cdot \frac{1}{4}=\frac{K_{2} N}{48 M_{1}}\left(1-\frac{M_{1}}{N}\right),
\end{aligned}
$$

where (a) follows from $x \geq\lfloor x\rfloor \geq \frac{x}{2}$ for any $x \geq 1$ and $\left|\frac{N-M_{1}}{2 M_{2}}\right| \leq \frac{N-M_{1}}{2 M_{2}} \leq K_{2} \leq \frac{K_{1} K_{2}}{K_{1}} \leq \frac{N}{2}$ by using $\frac{N-M_{1}}{2 K_{2}} \leq$ $M_{2}, N \geq K_{1} K_{2}$, and $K_{1} \geq 2$, (b) follows from $M_{2} \leq \frac{N-M_{1}}{K_{2}}$ and $\frac{M_{1}}{N} \geq \frac{N / 4}{N}=\frac{1}{4}$ by using $M_{1} \geq \frac{N}{4}$. Combining with (27), we have

$$
\begin{aligned}
& R_{1}^{l b}\left(M_{1}, M_{2}\right) \geq \frac{K_{2} N}{48 M_{1}}\left(1-\frac{M_{1}}{N}\right) \\
& \geq \frac{1}{48} \min \left\{K_{1} K_{2}, \frac{N}{M_{2}}\left(1-\frac{M_{2}}{N}\right), \frac{N K_{2}}{M_{1}+M_{2} K_{2}},\right. \\
& \left.\frac{K_{2} N}{M_{1}}\left(1-\frac{M_{1}}{N}\right)\right\} \geq \frac{1}{48} R_{1}^{u b}\left(M_{1}, M_{2}\right) .
\end{aligned}
$$

2) Gap between $R_{1}^{u b}\left(M_{1}, M_{2}\right)$ and $R_{1}^{l b}\left(M_{1}, M_{2}\right)$ in Subregime II: In this subregime, i.e., $M_{1}+K_{2} M_{2} \leq N$ and $\frac{N}{2} \leq M_{1} \leq N$, we have $M_{2} \leq \frac{N-M_{1}}{K_{2}}$. Then, we consider two regions:

A) $\frac{N}{2} \leq M_{1}<N$ and $0 \leq M_{2} \leq \frac{N-M_{1}}{2 K_{2}}$;

B) $\frac{N}{2} \leq M_{1}<N$ and $\frac{N-M_{1}}{2 K_{2}} \leq M_{2} \leq \frac{N-M_{1}}{K_{2}}$,

- A) $\frac{N}{2} \leq M_{1}<N$ and $0 \leq M_{2} \leq \frac{N-M_{1}}{2 K_{2}}$ : we choose $s_{1}=1$ and $s_{2}=K_{2}$ in the lower bound (17). Then, we have

$R_{1}^{l b}\left(M_{1}, M_{2}\right) \geq \frac{K_{2}\left(N-M_{1}-K_{2} M_{2}\right)}{N+K_{2}}$

$\stackrel{(a)}{\geq} \frac{K_{2}\left(N-M_{1}-\frac{N-M_{1}}{2}\right)}{N+N / 2}=\frac{K_{2}\left(N-M_{1}\right)}{3 N}=\frac{K_{2} N}{3 N}\left(1-\frac{M_{1}}{N}\right)$ $=\frac{K_{2} N}{6 \cdot N / 2}\left(1-\frac{M_{1}}{N}\right) \stackrel{(b)}{\geq} \frac{K_{2} N}{6 M_{1}}\left(1-\frac{M_{1}}{N}\right)$,

where $(a)$ follows from $M_{2} \leq \frac{N-M_{1}}{2 K_{2}}$ and $K_{2}=\frac{K_{1} K_{2}}{K_{1}} \leq$ $\frac{K_{1} K_{2}}{2} \leq \frac{N}{2}$ using $N>K_{1} K_{2}$ and $K_{1} \geq 2$, and (b) follows from $\frac{N}{2} \leq M_{1}$. Combining with (27), we have

$$
\begin{aligned}
& R_{1}^{l b}\left(M_{1}, M_{2}\right) \geq \frac{K_{2} N}{6 M_{1}}\left(1-\frac{M_{1}}{N}\right) \\
\geq & \frac{1}{6} \min \left\{K_{1} K_{2}, \frac{N}{M_{2}}\left(1-\frac{M_{2}}{N}\right), \frac{N K_{2}}{M_{1}+M_{2} K_{2}}, \frac{K_{2} N}{M_{1}}\left(1-\frac{M_{1}}{N}\right)\right\} \\
\geq & \frac{1}{6} R_{1}^{u b}\left(M_{1}, M_{2}\right) .
\end{aligned}
$$

- B) $\frac{N}{2} \leq M_{1}<N$ and $\frac{N-M_{1}}{2 K_{2}} \leq M_{2} \leq \frac{N-M_{1}}{K_{2}}$ : we choose $s_{1}=1$ and $s_{2}=\left\lfloor\frac{N-M_{1}}{2 M_{2}}\right\rfloor$ in the lower bound (17). This is a valid choice since

$$
1 \stackrel{(a)}{\leq}\left\lfloor\frac{N-M_{1}}{K_{2} M_{2}}\right\rfloor \stackrel{(b)}{\leq}\left\lfloor\frac{N-M_{1}}{2 M_{2}}\right\rfloor \stackrel{(c)}{\leq} \frac{N-M_{1}}{2 M_{2}} \stackrel{(d)}{\leq} K_{2},
$$

where (a) follows from $M_{2} \leq \frac{N-M_{1}}{K_{2}}$, (b) follows from $K_{2} \geq$ 2 , (c) follows from $x \geq\lfloor x\rfloor$ for any $x \geq 1$, and $(d)$ follows from $\frac{N-M_{1}}{2 K_{2}} \leq M_{2}$.

Substituting $s_{1}$ and $s_{2}$ in the lower bound (17), we obtain

$$
\begin{aligned}
& R_{1}^{l b}\left(M_{1}, M_{2}\right) \geq \frac{\left\lfloor\frac{N-M_{1}}{2 M_{2}}\right\rfloor\left(N-M_{1}-\left\lfloor\frac{N-M_{1}}{2 M_{2}}\right\rfloor M_{2}\right)}{N+\left\lfloor\frac{N-M_{1}}{2 M_{2}}\right\rfloor} \\
& \text { (a) } \frac{\frac{N-M_{1}}{4 M_{2}}\left(N-M_{1}-\frac{N-M_{1}}{2 M_{2}} M_{2}\right)}{N+\frac{N}{2}}=\frac{\left(N-M_{1}\right)^{2}}{12 M_{2} N} \\
& =\frac{K_{2} N}{12 M_{1}} \frac{N-M_{1}}{K_{2} M_{2}} \frac{M_{1}}{N}\left(1-\frac{M_{1}}{N}\right) \stackrel{(b)}{\geq} \frac{K_{2} N}{24 M_{1}}\left(1-\frac{M_{1}}{N}\right),
\end{aligned}
$$

where $(a)$ follows from $x \geq\lfloor x\rfloor \geq \frac{x}{2}$ for any $x \geq 1$, and $\left\lfloor\frac{N-M_{1}}{2 M_{2}}\right\rfloor \leq \frac{N-M_{1}}{2 M_{2}} \leq K_{2} \leq \frac{N}{K_{1}} \leq \frac{N}{2}$ using $\frac{N-M_{1}}{K_{2}} \leq M_{2}$, $N \geq K_{1} K_{2}$, and $K_{1} \geq 2$, and (b) follows from $\frac{N-M_{1}}{K_{2} M_{2}} \geq 1$ and $\frac{M_{1}}{N} \geq \frac{1}{2}$. Combining with (27), we have

$$
R_{1}^{l b}\left(M_{1}, M_{2}\right) \geq \frac{K_{2} N}{24 M_{1}}\left(1-\frac{M_{1}}{N}\right) \geq \frac{1}{24} R_{1}^{u b}\left(M_{1}, M_{2}\right) .
$$

\section{B. Gap between $R_{1}^{u b}\left(M_{1}, M_{2}\right)$ and $R_{1}^{l b}\left(M_{1}, M_{2}\right)$ in Regime} II

In regime II, we consider two subregimes, i.e., Subregime I) $0 \leq M_{1} \leq \frac{N}{2}$ and Subregime II) $\frac{N}{2} \leq M_{1} \leq N$. Then, we will discuss the gap in the two subregimes, respectively.

1) Gap in Subregime I: In this subregime, i.e., $M_{1}+$ $K_{2} M_{2} \geq N$ and $0 \leq M_{1} \leq \frac{N}{2}$, we have $M_{2} \geq \frac{N-M_{1}}{K_{2}} \geq$ $\frac{N}{2 K_{2}} \geq \frac{N}{4 K_{2}}$. Then, we consider:
A) $0 \leq M_{1}<\frac{N}{2 K_{1}}$ and $\frac{N}{2 K_{2}} \leq M_{2} \leq \frac{N}{2}$;
B) $0 \leq M_{1}<\frac{N}{2 K_{1}}$ and $\frac{N}{2} \leq M_{2} \leq N$;
C) $\frac{N}{2 K_{1}} \leq M_{1}<\frac{N}{4}$ and $\frac{N}{4 K_{2}} \leq M_{2} \leq \frac{N}{2}$;
D) $\frac{N}{2 K_{1}} \leq M_{1}<\frac{N}{4}$ and $\frac{N}{2} \leq M_{2} \leq N$;
E) $\frac{N}{4} \leq M_{1}<\frac{N}{2}$ and $\frac{N-M_{1}}{K_{2}} \leq M_{2} \leq \frac{N-M_{1}}{2}$;
F) $\frac{N}{4} \leq M_{1}<\frac{N}{2}$ and $\frac{N-M_{1}}{2} \leq M_{2} \leq N$.

- A) $0 \leq M_{1}<\frac{N}{2 K_{1}}$ and $\frac{N}{2 K_{2}} \leq M_{2} \leq \frac{N}{2}$ : we choose $s_{1}=1$ and $s_{2}=\left\lfloor\frac{N}{2 M_{2}}\right\rfloor$ in the lower bound (17). This is a valid choice since

$$
1 \leq\left\lfloor\frac{N}{2 \cdot N / 2}\right\rfloor \stackrel{(a)}{\leq}\left\lfloor\frac{N}{2 M_{2}}\right\rfloor \stackrel{(b)}{\leq} \frac{N}{2 M_{2}} \stackrel{(c)}{\leq} K_{2},
$$

where ( $a$ ) follows from $M_{2} \leq \frac{N}{2}$, (b) follows from $x \geq\lfloor x\rfloor$ for any $x \geq 1$, and $(c)$ follows from $\frac{N}{2 K_{2}} \leq M_{2}$.

Substitute $s_{1}$ and $s_{2}$ into the lower bound (17), we have

$$
\begin{aligned}
& R_{1}^{l b}\left(M_{1}, M_{2}\right) \geq \frac{\left\lfloor\frac{N}{2 M_{2}}\right\rfloor\left(N-M_{1}-\left\lfloor\frac{N}{2 M_{2}}\right\rfloor M_{2}\right)}{N+\left\lfloor\frac{N}{2 M_{2}}\right\rfloor} \\
& \text { (a) } \frac{\frac{N}{4 M_{2}}\left(N-M_{1}-\frac{N}{2}\right)}{N+\frac{N}{2 M_{2}}}=\frac{\frac{N}{4 M_{2}}\left(\frac{1}{2}-\frac{M_{1}}{N}\right)}{1+\frac{1}{2 M_{2}}} \\
& \stackrel{\text { (b) }}{\geq} \frac{\frac{N}{4 M_{2}}\left(\frac{1}{2}-\frac{1}{4}\right)}{1+\frac{1}{2}}=\frac{N}{24 M_{2}} \geq \frac{N}{24 M_{2}}\left(1-\frac{M_{2}}{N}\right) \text {, }
\end{aligned}
$$

where ( $a$ ) follows from $x \geq\lfloor x\rfloor \geq \frac{x}{2}$ for any $x \geq 1$, (b) follows from $\frac{M_{1}}{N} \leq \frac{N /\left(2 K_{1}\right)}{N}=\frac{1}{2 K_{1}} \leq \frac{1}{4}$ using $M_{1} \leq \frac{1}{2 K_{1}}$ 
and $K_{1} \geq 2$, and $\frac{1}{2 M_{2}} \leq \frac{K_{2}}{N} \leq \frac{K_{1} K_{2}}{K_{1} N} \leq \frac{1}{K_{1}} \leq \frac{1}{2}$ using $\frac{N}{2 K_{2}} \leq M_{2}, N \geq K_{1} K_{2}$, and $K_{1} \geq 2$. Combining with (27), we have

$$
R_{1}^{l b}\left(M_{1}, M_{2}\right) \geq \frac{N}{24 M_{2}}\left(1-\frac{M_{2}}{N}\right) \geq \frac{1}{24} R_{1}^{u b}\left(M_{1}, M_{2}\right) .
$$

- B) $0 \leq M_{1}<\frac{N}{2 K_{1}}$ and $\frac{N}{2} \leq M_{2} \leq N$ : We have

$$
R_{1}^{l b} \geq 0 \geq \frac{N}{M_{2}}-2=\frac{N}{M_{2}}\left(1-\frac{M_{2}}{N}\right)-1 .
$$

Combining with (31), we have

$$
R_{1}^{l b}\left(M_{1}, M_{2}\right) \geq \frac{N}{M_{2}}\left(1-\frac{M_{2}}{N}\right)-1 \geq R_{1}^{u b}\left(M_{1}, M_{2}\right)-1 .
$$

- C) $\frac{N}{2 K_{1}} \leq M_{1}<\frac{N}{4}$ and $\frac{N}{4 K_{2}} \leq M_{2} \leq \frac{N}{2}$ : Let

$$
\left(s_{1}, s_{2}\right)= \begin{cases}\left(\left\lfloor\frac{N}{4 M_{1}}\right\rfloor,\left\lfloor\frac{M_{1}}{M_{2}}\right\rfloor\right), & \text { if } M_{1} \geq M_{2}, \\ \left(\left\lfloor\frac{N}{4 M_{2}}\right\rfloor, 1\right), & \text { otherwise. }\end{cases}
$$

in the lower bound (17). This is a valid choice since for $M_{1} \geq$ $M_{2}$, we have

$$
1=\left\lfloor\frac{N}{4 \cdot N / 4}\right\rfloor \stackrel{(a)}{\leq}\left\lfloor\frac{N}{4 M_{1}}\right\rfloor \stackrel{(b)}{\leq} \frac{N}{4 M_{1}} \stackrel{(c)}{\leq} \frac{K_{1}}{2}
$$

and

$$
1=\left\lfloor\frac{M_{1}}{M_{2}}\right\rfloor \leq \frac{M_{1}}{M_{2}} \stackrel{(d)}{\leq} \frac{N / 4}{N /\left(4 K_{2}\right)}=K_{2},
$$

where (a) follows from $M_{1} \geq \frac{N}{4}$, (b) follows from $x \geq\lfloor x\rfloor \geq$ $\frac{x}{2}$ for any $x \geq 1,(c)$ follows from $M_{1} \geq \frac{N}{2 K_{1}},(d)$ follows from $M_{1} \leq \frac{N}{4}$ and $M_{2} \geq \frac{N}{4 K_{2}}$.

For $M_{1}<M_{2}$, we have

$$
1=\left\lfloor\frac{N}{4 \cdot N / 4}\right\rfloor \leq\left\lfloor\frac{N}{4 M_{2}}\right\rfloor \leq\left\lfloor\frac{N}{4 M_{1}}\right\rfloor \leq \frac{N}{4 M_{1}} \stackrel{(a)}{\leq} \frac{K_{1}}{2},
$$

where (a) follows from $M_{1} \geq \frac{N}{2 K_{1}}$.

Note that $s_{1} \leq \frac{N}{4 M_{1}}$ and $\frac{N}{16 M_{2}} \leq s_{1} s_{2} \leq \frac{N}{4 M_{2}}$ due to $x \geq\lfloor x\rfloor \geq \frac{x}{2}$ for any $x \geq 1$. Then, we have

$$
\begin{aligned}
& R_{1}^{l b}\left(M_{1}, M_{2}\right) \geq \frac{\frac{N}{16 M_{2}}\left(N-\frac{N}{4 M_{1}} M_{1}-\frac{N}{4 M_{2}} M_{2}\right)}{N+\frac{N}{4 M_{2}}} \\
& \stackrel{(a) \frac{N}{16 M_{2}}\left(N-\frac{N}{2}\right)}{N+\frac{N}{2}}=\frac{N}{48 M_{2}} \geq \frac{N}{48 M_{2}}\left(1-\frac{M_{2}}{N}\right),
\end{aligned}
$$

where $(a)$ follows from $\frac{N}{4 M_{2}} \leq K_{2}=\frac{K_{1} K_{2}}{K_{1}} \leq \frac{K_{1} K_{2}}{2} \leq \frac{N}{2}$ using $N>K_{1} K_{2}$ and $K_{1} \geq 2$. Combined with 27), we have

$$
R_{1}^{l b}\left(M_{1}, M_{2}\right) \geq \frac{N}{48 M_{2}}\left(1-\frac{M_{2}}{N}\right) \geq \frac{1}{48} R_{1}^{u b}\left(M_{1}, M_{2}\right) .
$$

- D) $\frac{N}{2 K_{1}} \leq M_{1}<\frac{N}{4}$ and $\frac{N}{2} \leq M_{2} \leq N$ : We have

$$
R_{1}^{l b}\left(M_{1}, M_{2}\right) \geq 0 \geq \frac{N}{M_{2}}-2 \geq \frac{N}{M_{2}}\left(1-\frac{M_{2}}{N}\right)-1 .
$$

Combined with (31), we have

$$
R_{1}^{l b}\left(M_{1}, M_{2}\right) \geq \frac{N}{M_{2}}\left(1-\frac{M_{2}}{N}\right)-1 \geq R_{1}^{u b}\left(M_{1}, M_{2}\right)-1 .
$$

- E) $\frac{N}{4} \leq M_{1}<\frac{N}{2}$ and $\frac{N-M_{1}}{K_{2}} \leq M_{2} \leq \frac{N-M_{1}}{2}$ : we choose $s_{1}=1$ and $s_{2}=\left\lfloor\frac{N-M_{1}}{2 M_{2}}\right\rfloor$ in the lower bound (17). This is a valid choice since

$$
1 \leq\left\lfloor\frac{N-M_{1}}{2 M_{2}}\right\rfloor \leq \frac{N-M_{1}}{2 M_{2}} \stackrel{(a)}{\leq} \frac{K_{2}}{2},
$$

where $(a)$ follows from $\frac{N-M_{1}}{K_{2}} \leq M_{2}$.

Substitute $s_{1}$ and $s_{2}$ into the lower bound (17), we have

$$
\begin{aligned}
R_{1}^{l b}\left(M_{1}, M_{2}\right) & \geq \frac{\left\lfloor\frac{N-M_{1}}{2 M_{2}}\right\rfloor\left(N-M_{1}-\left\lfloor\frac{N-M_{1}}{2 M_{2}}\right\rfloor M_{2}\right)}{N+\left\lfloor\frac{N-M_{1}}{2 M_{2}}\right\rfloor} \\
& \geq \frac{(a) \frac{N-M_{1}}{4 M_{2}}\left(N-M_{1}-\frac{N-M_{1}}{2}\right)}{N+\frac{N}{4}} \\
& =\frac{\left(N-M_{1}\right)^{2}}{10 N M_{2}},
\end{aligned}
$$

where $(a)$ follows from $x \geq\lfloor x\rfloor \geq \frac{x}{2}$ for any $x \geq 1$, and $\left|\frac{N-M_{1}}{2 M_{2}}\right| \leq\left\lfloor\frac{K_{2}}{2}\right\rfloor \leq \frac{K_{2}}{2} \leq \frac{K_{1} K_{2}}{2 K_{1}} \leq \frac{N}{4}$ using $\frac{N-M_{1}}{K_{2}} \leq M_{2}$, $K_{1} K_{2} \leq N$, and $K_{1} \geq 2$. Combining with (27), we have

$$
\begin{aligned}
& R_{1}^{l b}\left(M_{1}, M_{2}\right) \geq \frac{\left(N-M_{1}\right)^{2}}{10 N M_{2}} \geq \frac{1}{20} \min \left\{K_{1} K_{2}, \frac{N}{M_{2}}\left(1-\frac{M_{2}}{N}\right),\right. \\
& \left.\frac{2\left(N-M_{1}\right)^{2}}{N M_{2}}\right\} \geq \frac{1}{20} R_{1}^{u b}\left(M_{1}, M_{2}\right) .
\end{aligned}
$$

- F) $\frac{N}{4} \leq M_{1}<\frac{N}{2}$ and $\frac{N-M_{1}}{2} \leq M_{2} \leq N$ : We have

$$
\begin{aligned}
& R_{1}^{l b}\left(M_{1}, M_{2}\right) \geq 0=\frac{2\left(N-M_{1}\right)^{2}}{N M_{2}}-\frac{2\left(N-M_{1}\right)^{2}}{N M_{2}} \\
& =\frac{2\left(N-M_{1}\right)^{2}}{N M_{2}}-\frac{N-M_{1}}{M_{2}} \cdot \frac{2\left(N-M_{1}\right)}{N} \\
& \stackrel{(a)}{\geq} \frac{2\left(N-M_{1}\right)^{2}}{N M_{2}}-2 \cdot 2=\frac{2\left(N-M_{1}\right)^{2}}{N M_{2}}-4,
\end{aligned}
$$

where $(a)$ follows from $\frac{N-M_{1}}{2} \leq M_{2}$ and $\frac{N-M_{1}}{N} \leq 1$.

Combined with (31), we have

$$
\begin{aligned}
& R_{1}^{l b}\left(M_{1}, M_{2}\right) \geq \frac{2\left(N-M_{1}\right)^{2}}{N M_{2}}-4 \geq \min \left\{K_{1} K_{2},\right. \\
& \left.\frac{N}{M_{2}}\left(1-\frac{M_{2}}{N}\right), \frac{2\left(N-M_{1}\right)^{2}}{N M_{2}}\right\}-4 \geq R_{1}^{u b}\left(M_{1}, M_{2}\right)-4 .
\end{aligned}
$$

2) Gap in Subregime II: In this subregime, i.e., $M_{1}+$ $K_{2} M_{2} \geq N$ and $\frac{N}{2} \leq M_{1} \leq N$, we have $M_{2} \geq \frac{N-M_{1}}{K_{2}}$. Then, we consider:

A) $\frac{N}{2} \leq M_{1}<N$ and $\frac{N-M_{1}}{K_{2}} \leq M_{2} \leq \frac{N-M_{1}}{2}$;

B) $\frac{N}{2} \leq M_{1}<N$ and $\frac{N-M_{1}}{2} \leq M_{2} \leq N$.

- A) $\frac{N}{2} \leq M_{1}<N$ and $\frac{N-M_{1}}{K_{2}} \leq M_{2} \leq \frac{N-M_{1}}{2}$ : we choose $s_{1}=1$ and $s_{2}=\left\lfloor\frac{N-M_{1}}{2 M_{2}}\right\rfloor$ in the lower bound (17). This is a valid choice since

$$
1 \leq\left\lfloor\frac{N-M_{1}}{2 M_{2}}\right\rfloor \stackrel{(a)}{\leq} \frac{N-M_{1}}{2 M_{2}} \stackrel{(b)}{\leq} \frac{K_{2}}{2},
$$

where $(a)$ follows from $x \geq\lfloor x\rfloor$ for any $x \geq 1$, (b) follows from $\frac{N-M_{1}}{K_{2}} \leq M_{2}$. 
Then, we obtain

$$
\begin{aligned}
R_{1}^{l b}\left(M_{1}, M_{2}\right) & \geq \frac{\left\lfloor\frac{N-M_{1}}{2 M_{2}}\right\rfloor\left(N-M_{1}-\left\lfloor\frac{N-M_{1}}{2 M_{2}}\right\rfloor M_{2}\right)}{N+\left\lfloor\frac{N-M_{1}}{2 M_{2}}\right\rfloor} \\
& \stackrel{(a)}{\geq} \frac{\frac{N-M_{1}}{4 M_{2}}\left(N-M_{1}-\frac{N-M_{1}}{2 M_{2}} M_{2}\right)}{N+\frac{N}{4}} \\
& =\frac{\left(N-M_{1}\right)^{2}}{10 M_{2} N},
\end{aligned}
$$

where (a) follows from $x \geq\lfloor x\rfloor \geq \frac{x}{2}$ for any $x \geq 1$ and $\left.\mid \frac{N-M_{1}}{2 M_{2}}\right\rfloor \leq\left\lfloor\frac{K_{2}}{2}\right\rfloor \leq \frac{K_{2}}{2} \leq \frac{K_{1} K_{2}}{2 K_{1}} \leq \frac{N}{4}$ using $\frac{N-M_{1}}{K_{2}} \leq M_{2}$, $N \geq K_{1} K_{2}$, and $K_{1} \geq 2$. Combining with (31), we have

$$
R_{1}^{l b}\left(M_{1}, M_{2}\right) \geq \frac{\left(N-M_{1}\right)^{2}}{10 M_{2} N} \geq \frac{1}{20} R_{1}^{u b}\left(M_{1}, M_{2}\right) .
$$

- B) $\frac{N}{2} \leq M_{1}<N$ and $\frac{N-M_{1}}{2} \leq M_{2} \leq N$ : We have

$$
\begin{aligned}
& R_{1}^{l b}\left(M_{1}, M_{2}\right) \geq 0=\frac{2\left(N-M_{1}\right)^{2}}{N M_{2}}-\frac{2\left(N-M_{1}\right)^{2}}{N M_{2}} \\
& =\frac{2\left(N-M_{1}\right)^{2}}{N M_{2}}-\frac{N-M_{1}}{M_{2}} \cdot \frac{2\left(N-M_{1}\right)}{N} \\
& \stackrel{(a)}{\geq} \frac{2\left(N-M_{1}\right)^{2}}{N M_{2}}-2 \cdot 2=\frac{2\left(N-M_{1}\right)^{2}}{M_{2} N}-4,
\end{aligned}
$$

where (a) follows from $\frac{N-M_{1}}{2} \leq M_{2}$ and $\frac{N-M_{1}}{N} \leq 1$.

Combining with (31), we have

$$
\left.R_{1}^{l b} M_{1}, M_{2}\right) \geq \frac{2\left(N-M_{1}\right)^{2}}{M_{2} N}-4 \geq R_{1}^{u b}\left(M_{1}, M_{2}\right)-4 .
$$

Combining the results in Subsections A and B, we have that the upper bound $R_{1}^{u b}$ and the lower bound $R_{1}^{l b}$ are within a constant multiplicative and additive gap for all pairs of $M_{1}$ and $M_{2}$. More specifically, we have

$$
R_{1}^{l b}\left(M_{1}, M_{2}\right) \geq \frac{1}{48} R_{1}^{u b}\left(M_{1}, M_{2}\right)-4 .
$$

\section{APPENDIX C \\ GAP BETWEEN THE UPPER BOUND $R_{2}^{u b}\left(M_{2}\right)$ AND LOWER BOUND $R_{2}^{l b}\left(M_{2}\right)$}

In this section, we will characterize the gap between the upper bound $R_{2}^{u b}\left(M_{2}\right)$ and the lower bound $R_{2}^{l b}\left(M_{2}\right)$. We also consider $K_{1} \geq 2, K_{1} \geq 2$, and $N \geq K_{1} K_{2}$. Recall that lower bound and upper bound of the transmission rate $R_{2}\left(\alpha^{*}, \beta^{*}\right)$ are

$$
R_{2}^{l b}\left(M_{2}\right) \triangleq \max _{t \in\left[K_{2}\right]} \frac{t\left(N-t M_{2}\right)}{N+t}
$$

and

$$
R_{2}^{u b}\left(M_{2}\right)=2 \min \left\{K_{2}, \frac{N}{M_{2}}\right\},
$$

respectively.

To discuss the gap between the lower and the upper bound, we consider two regimes:

A) $0 \leq M_{2}<\frac{N}{2}$;

B) $\frac{N}{2} \leq M_{2} \leq N$.
- A) $0 \leq M_{2}<\frac{N}{2}$ : we choose $t=\left\lfloor\frac{1}{2} \min \left\{K_{2}, \frac{N}{M_{2}}\right\}\right\rfloor$ in the lower bound (84). This is a valid choice since

$$
1=\left\lfloor\frac{1}{2} \min \left\{K_{2}, \frac{N}{M_{2}}\right\}\right\rfloor \leq \frac{K_{2}}{2} .
$$

Then, we have

$$
\begin{aligned}
& R_{2}^{l b}\left(M_{2}\right) \geq \frac{\left\lfloor\frac{1}{2} \min \left\{K_{2}, \frac{N}{M_{2}}\right\}\right\rfloor\left(N-\left\lfloor\frac{1}{2} \min \left\{K_{2}, \frac{N}{M_{2}}\right\}\right\rfloor M_{2}\right)}{N+\left\lfloor\frac{1}{2} \min \left\{K_{2}, \frac{N}{M_{2}}\right\}\right\rfloor} \\
& \text { (a) } \frac{\frac{1}{4} \min \left\{K_{2}, \frac{N}{M_{2}}\right\}\left(N-\frac{N}{2}\right)}{N+\frac{N}{4}} \\
& =\frac{1}{10} \min \left\{K_{2}, \frac{N}{M_{2}}\right\},
\end{aligned}
$$

where (a) follows from $x \geq\lfloor x\rfloor \geq \frac{x}{2}$ for any $x \geq 1$ and $\left|\frac{1}{2} \min \left\{K_{2}, \frac{N}{M_{2}}\right\}\right| \leq \frac{K_{2}}{2} \leq \frac{K_{1} K_{2}}{2 K_{1}} \leq \frac{N}{4}$ using $N \geq K_{1} K_{2}$ and $K_{1} \geq 2$. Combining with $[85$, we have

$$
\begin{aligned}
& R_{2}^{l b}\left(M_{2}\right) \geq \frac{1}{10} \min \left\{K_{2}, \frac{N}{M_{2}}\right\} \geq \frac{1}{20} \cdot 2 \min \left\{K_{2}, \frac{N}{M_{2}}\right\} \\
& \geq \frac{1}{20} R_{2}^{u b}\left(M_{2}\right) .
\end{aligned}
$$

- B) $\frac{N}{2} \leq M_{2}<N$ : We have

$$
R_{2}^{l b}\left(M_{2}\right) \geq 0=2 \frac{N}{M_{2}}-2 \frac{N}{M_{2}} .
$$

Combining with $(85)$, we have

$$
\begin{aligned}
& R_{2}^{l b}\left(M_{2}\right) \geq 2 \frac{N}{M_{2}}-2 \frac{N}{M_{2}} \geq 2 \min \left\{K_{2}, \frac{N}{M_{2}}\right\}-4 \\
& \geq R_{2}^{u b}\left(M_{2}\right)-4 .
\end{aligned}
$$

By combining (88) and (90), we have

$$
R_{2}^{l b}\left(M_{2}\right) \geq \frac{1}{20} R_{2}^{u b}\left(M_{2}\right)-4 .
$$

\section{REFERENCES}

[1] L. Zhang, Z. Wang, M. Xiao, and G. Wu, "Order-optimal caching in hierarchical networks," in IEEE/CIC International Conference on Communications in China (ICCC), 2016, pp. 1-5.

[2] L. Zhang, M. Xiao, G. Wu, M. Alam, Y.-C. Liang, and S. Li, "A survey of advanced techniques for spectrum sharing in $5 \mathrm{~g}$ networks," IEEE Wireless Commun., vol. 65, no. 10, pp. 4152-4162, Oct. 2017.

[3] G. Yang, Y.-C. Liang, R. Zhang, , and Y. Pei, "Modulation in the air: Backscatter communications over ambient ofdm signals," IEEE Trans. Commun., vol. 66, no. 3, pp. 1219-1233, Mar. 2018.

[4] G. Yang, Q. Zhang, and Y.-C. Liang, "Cooperative ambient backscatter communication systems for future internet-of-things," IEEE Internet Things J., vol. 5, no. 2, pp. 1116-1130, Apr. 2018.

[5] X. Kang, Y.-C. Liang, and J. Yang, "Riding on the primary: A new spectrum sharing paradigm for wireless-powered iot devices," IEEE Trans. on Wireless Commun., vol. 17, no. 9, pp. 6335-6347, Sep. 2018.

[6] L. Zhang, J. Liu, M. Xiao, G. Wu, Y.-C. Liang, and S. Li, "Performance analysis and optimization in downlink noma systems with cooperative full-duplex relaying," IEEE J. Sel. Areas in Commun., vol. 35, no. 10, pp. 2398-2412, Oct. 2017.

[7] L. W. Dowdy and D. V. Foster, "Comparative models of the file assignment problem," ACM Computing Survey, vol. 14, no. 2, pp. 287313, Jun. 1982.

[8] M. R. Korupolu, C. G. Plaxton, and R. Rajaraman, "Placement algorithms for hierarchical cooperative caching," Journal of Algorithms, vol. 38, no. 1, pp. 260-302, 2001.

[9] J. Kangasharju, J. W. Roberts, and K. W. Ross, "Object replication strategies in content distribution networks," Сотр. Сотти. Journal, vol. 25, no. 4, pp. 376-383, Mar. 2002. 
[10] I. D. Baev, R. Rajaraman, and C. Swamy, "Approximating algorithms for data placement problems," SIAM J. Computing, vol. 38, no. 4, pp. 1411-1429, 2008.

[11] S. Borst, V. Gupta, and A. Walid, "Distributed caching algorithms for content distribution networks," in Proceedings of IEEE INFOCOM, 2010.

[12] M. A. Maddah-Ali and U. Niesen, "Fundamental limits of caching," IEEE Trans. Inform. Theory, vol. 60, no. 5, pp. 2856-2867, May 2014.

[13] — "Decentralized coded caching attains order-optimal memory-rate tradeoff," IEEE/ACM Trans. Netw., vol. 23, no. 4, pp. 1029-1040, 2015.

[14] N. Karamchandani, U. Niesen, M. A. Maddah-Ali, and S. Diggavi, "Hierarchical coded caching," IEEE Trans. Inform. Theory, vol. 62, no. 6, pp. 3212-3229, Jun. 2016.

[15] H. Ghasemi and A. Ramamoorthy, "Improved lower bounds for coded caching," IEEE Trans. Inform. Theory, vol. 63, no. 7, pp. 4388-4413, Jul. 2017.

[16] M. M. Amiri and D. Gndz, "Fundamental limits of coded caching: improved delivery rate-cache capacity tradeoff," IEEE Trans. Commun., vol. 65, no. 2, pp. 806-815, Feb. 2017.

[17] M. M. Amiri, Q. Yang, and D. Gndz, "Decentralized coded caching with distinct cache capacities," in 50th Asilomar Conference on Signals, Systems and Computers, 2016, pp. 734-738.

[18] A. M. Ibrahim, A. A. Zewail, and A. Yener, "Centralized coded caching with heterogeneous cache sizes," in IEEE Wireless Communications and Networking Conference (WCNC), 2017, pp. 1-6.

[19] U. Niesen and M. A. Maddah-Ali, "Coded caching with nonuniform demands," IEEE Trans. Inform. Theory, vol. 63, no. 2, pp. 1146-1158, Feb. 2017.

[20] M. Ji, A. M. Tulino, J. Llorca, and G. Caire, "Order-optimal rate of caching and coded multicasting with random demands," IEEE Trans. Inform. Theory, vol. 63, no. 6, pp. 3923-3949, Jun. 2017.

[21] J. Zhang, X. Lin, C.-C. Wang, and X. Wang, "Coded caching for files with distinct file sizes," in IEEE International Symposium on Information Theory (ISIT), 2015, pp. 1686-1690.

[22] M. Ji, A. M. Tulino, J. Llorca, and G. Caire, "Caching and coded multicasting: Multiple groupcast index coding," in Proc. IEEE Global Conf. Signal and Information Processing (GlobalSIP), Dec. 2014, pp. 881-885.

[23] M. Ji, A. Tulino, J. Llorca, and G. Caire, "Caching-aided coded multicasting with multiple random requests," in IEEE Information Theory Workshop $(I T W), 2015$, pp. 1-5.

[24] A. Sengupta and R. Tandon, "Improved approximation of storage-rate tradeoff for caching with multiple demands," IEEE Trans. Commun., vol. 65, no. 5, pp. 1940-1955, May 2017.

[25] R. Pedarsani, M. A. Maddah-Ali, and U. Niesen, "Online coded caching," IEEE/ACM Trans. Netw., vol. 24, no. 2, pp. 836-845, Apr. 2016.

[26] Q. Yan, U. Parampalli, X. Tang, and Q. Chen, "Online coded caching with random access," IEEE Commu. Letters, vol. 21, no. 3, pp. 552-555, Mar. 2017.

[27] J. Hachem, N. Karamchandani, and S. N. Diggavi, "Coded caching for multi-level popularity and access," IEEE Trans. Inform. Theory, vol. 63, no. 5, pp. 3108-3141, May 2017.

[28] X. Xu and M. Tao, "Modeling, analysis, and optimization of coded caching in small-cell networks," To appear in IEEE Trans. Commun., 2017.

[29] M. Ji, G. Caire, and A. F. Molisch, "Fundamental limits of caching in wireless d2d networks," IEEE Trans. Inform. Theory, vol. 62, no. 2, pp. 849-869, Feb. 2014.

[30] L. Zhang, M. Xiao, G. Wu, and S. Li, "Efficient scheduling and power allocation for $\mathrm{d} 2 \mathrm{~d}$-assisted wireless caching networks," IEEE Trans. Commun., vol. 64, no. 6, pp. 2438-2452, Jun. 2016.

[31] K. Shanmugam, M. Ji, A. M. Tulino, J. Llorca, and A. G. Dimakis., "Finite-length analysis of caching-aided coded multicasting," IEEE Trans. Inform. Theory, vol. 62, no. 10, pp. 5524-5537, Oct. 2016.

[32] K. Shanmugam, A. M. Tulino, and A. G. Dimakis, "Coded caching with linear subpacketization is possible using ruzsa-szem éredi graphs," in IEEE International Symposium on Information Theory (ISIT), Jun. 2017, pp. 1237-1241.

[33] L. Tang and A. Ramamoorthy, "Low subpacketization schemes for coded caching," in IEEE International Symposium on Information Theory (ISIT), Jun. 2017, pp. 2790-2794.

[34] A. Sengupta, R. Tandon, and T. C. Clancy, "Fundamental limits of caching with secure delivery," IEEE Trans. Inf. Forensics Security, vol. 10, no. 2, pp. 355-370, Feb. 2015.
[35] V. Ravindrakumar, P. Panda, N. Karamchandani, and V. Prabhakaran, "Fundamental limits of secretive coded caching," in IEEE International Symposium on Information Theory (ISIT), 2016, pp. 425-429.

[36] F. Gabry, V. Bioglio, and I. Land, "On edge caching with secrecy constraints," in IEEE International Conference on Communications (ICC), 2016, pp. 1-6.

[37] A. E. Gamal and Y. H. Kim, Network Information Theory. Cambridge University Press, 2011. 